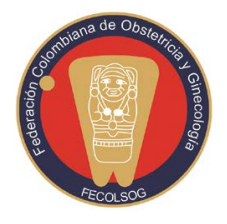

Revista Colombiana de Obstetricia y Ginecología Vol. 67 No. 2 • Abril-Junio 2016 • (120-146)

\title{
MANEJO QUIRÚRGICO DEL CÁNCER EPITELIAL DE OVARIO: GUIIA DE PRÁCTICA CLÍNICA DE LA ASOCIACIÓN COLOMBIANA DE GINECÓLOGOS ONCÓLOGOS (ASCGO)
}

\section{Surgical management of epithelial ovarian cancer: Clinical Practice Guideline of the Colombian Association of Gynaecologic Oncologists (ASCGO)}

\section{Grupo Desarrollador de la Guía*}

Recibido: marzo 14/16 - Aceptado: junio 9/16

\section{RESUMEN}

Introducción: el cáncer epitelial de ovario constituye la primera causa de muerte por cáncer ginecológico a nivel mundial. En Colombia cada año se presentan 8,7 casos nuevos por cada $100.000 \mathrm{mu}$ jeres y se registran más de 800 muertes por causa de esta enfermedad. La cirugía constituye el pilar de manejo de las pacientes con cáncer epitelial de ovario, su realización oportuna y la adecuada combinación con otras estrategias terapéuticas busca erradicar la enfermedad o, en los estados avanzados, lograr volúmenes tumorales microscópicos para mejorar el pronóstico. El objetivo de este trabajo consiste en desarrollar recomendaciones basadas en la evidencia para el diagnóstico y tratamiento quirúrgico integral del cáncer epitelial de ovario en población colombiana.

Materiales y métodos: guía de práctica clínica basada en la evidencia, desarrollada por un panel de expertos de la Asociación Colombiana de Ginecólogos Oncólogos. La Sociedad definió y validó un total de 33 preguntas de interés para el manejo quirúrgico integral. Se llevó a cabo una revisión de literatura de guías de práctica clínica y se actualizó la evidencia de las guías fuente usando revisiones de literatura y estudios primarios experimentales y observacionales acordes con la naturaleza de cada pregunta. Finalmente, se discutieron y validaron las recomendaciones en la Asociación mediante un método participativo tipo panel de expertos.

Resultados: se resumen las recomendaciones basadas en la mejor evidencia disponible para la detección temprana y el diagnóstico del cáncer epitelial de ovario, el manejo quirúrgico en estados tempranos y avanzados de la enfermedad, el seguimiento y manejo de las recaídas, así como el manejo con intención paliativa.

Conclusiones: esta GPC fue desarrollada utilizando procedimientos sistemáticos y la mejor evidencia disponible. Se espera que tenga un impacto positivo en el manejo y los desenlaces de las pacientes que padecen de esta enfermedad.

Palabras clave: neoplasias ováricas, diagnóstico, procedimientos quirúrgicos de citorreducción, Guía de Práctica Clínica.

\section{ABSTRACT}

Introduction: Epithelial ovarian cancer is the primary cause of death from gynaecological cancer in the world. In Colombia, every year there are 8.7 new cases for 100,000 women and more than 800 deaths as a result of this disease. Surgery is the 
mainstay for management of patients with epithelial ovarian cancer. The aim, when performed in a timely fashion and in combination with the right therapeutic strategies, is to eradicate the disease or, in advanced stages, to achieve microscopic tumour volumes in order to improve prognosis. The objective of this work is to develop evidencebased recommendations for the diagnosis and comprehensive surgical treatment of epithelial ovarian cancer in the Colombian population.

Materials and methods: Evidence-based clinical practice guideline developed by an expert panel from the Colombian Association of Gynaecologic Oncologists. A total of 33 questions of interest for comprehensive surgical management were defined and validated in the Society. A review of the literature on clinical practice guidelines was conducted. The evidence of the source guidelines was updated using reviews of the literature and primary experimental and observational studies according to the nature of each question. Finally, the recommendations were discussed and validated in the Association using a participatory expert panel method.

Results: A summary of recommendations of the best available evidence for early detection and diagnosis of epithelial ovarian cancer, surgical management of early and late stages of the disease, follow-up and management of recurrences, as well as management with palliative goals.

Conclusions: This CPG was developed using systematic procedures and the best available evidence. It is expected to have a positive impact on management and outcomes of patients suffering from this disease.

Key words: Ovarian neoplasms, diagnosis, cytoreductive surgical procedure, Clinical Practice Guideline.

\section{OBJETIVO Y ALCANCE DE LA GUÍA}

Formular y difundir recomendaciones basadas en la evidencia para el diagnóstico de masas anexiales y el tratamiento quirúrgico del cáncer epitelial de ovario en mujeres mayores de 18 años en Colombia, como estrategia para mejorar la calidad en la atención, reducir la variabilidad en el manejo de la entidad y y disminuir la mortalidad en estas pacientes.

La guía valora aspectos desde el diagnóstico de masas anexiales hasta algunos del manejo paliativo del cáncer epitelial de ovario (CEO), por tanto, los usuarios son médicos generales, ginecólogos y ginecólogos oncólogos.

Las recomendaciones formuladas en el documento se dirigen a dos poblaciones específicas:

- Mujeres mayores de 18 años con masa anexial en proceso diagnóstico.

- Mujeres mayores de 18 años con diagnóstico de cáncer epitelial de ovario en cualquier estadio.

\section{INTRODUCCIÓN}

El cáncer epitelial de ovario ocupa el cuarto puesto en frecuencia entre todas las enfermedades malignas de las mujeres después de los cánceres de mama, cuello uterino y endometrio, sin embargo, constituye la primera causa de muerte por cáncer ginecológico en el mundo (1). En Colombia cada año se presentan 8,7 casos nuevos por cada $100.000 \mathrm{mu}-$ jeres (2) y se estima que alrededor de 800 muertes anuales se producen a causa de la enfermedad (3).

Esta neoplasia se comporta como una enfermedad heterogénea, que incluye varios tipos histológicos, con distintos grados de diferenciación (1). El cáncer epitelial representa el 90\% de todas las neoplasias malignas del ovario en población general (4), siendo el tipo histológico más frecuente el seroso papilar, seguido por los tipos mucinoso, endometrioide, de células claras, transicional, mixto y los tumores indiferenciados (5).

A pesar de que la supervivencia a 5 años en los estadios tempranos (FIGO I y II) (tabla 1) es superior al $80 \%$ en comparación con los estadios más tardíos (FIGO III y IV) (tabla 1), la mortalidad global de la enfermedad es elevada, debido a que cerca del $70 \%$ de las pacientes se diagnostican en 
estadios avanzados (6). Esto se debe principalmente a que los estadios tempranos son asintomáticos o se manifiestan con síntomas inespecíficos, lo que hace que la enfermedad pase desapercibida en sus estadios iniciales, y a las dificultades existentes para un adecuado diagnóstico precoz (7).

Aunque el impacto de la quimioterapia en la supervivencia de la enfermedad ha sido sustancial, la evolución de las técnicas quirúrgicas en la cirugía de citorreducción primaria con el fin de lograr volúmenes tumorales microscópicos mínimos es el factor pronóstico más importante (8). El uso de abordajes quirúrgicos ultrarradicales ha cobrado importancia en el manejo de las pacientes (9), así como su combinación con regímenes de quimioterapia (10), para lograr mejores resultados en términos de supervivencia. Otras consideraciones importantes proceden del manejo quirúrgico de los estadios tempranos, en especial las relacionadas con la preservación de la fertilidad, el manejo conservador, así como el papel de la cirugía mínimamente invasiva.

El presente documento resume un conjunto de recomendaciones basadas en la evidencia y formuladas por un panel de expertos de la Asociación Colombiana de Ginecólogos Oncólogos, sobre las alternativas diagnósticas y terapéuticas para el manejo quirúrgico integral del cáncer epitelial de ovario (CEO). La revisión detallada de la evidencia se describe con mayor detalle en la Guía de Práctica Clínica para el manejo quirúrgico del cáncer epitelial de ovario (11).

\section{MATERIALES Y MÉTODOS}

Se realizó un proceso de adaptación y actualización de guías, con formulación de recomendaciones mediante un panel de expertos integrado por especialistas seleccionados por la Asociación Colombiana de Ginecólogos Oncólogos (ASCGO). En una primera instancia se definió el grupo desarrollador de la guía, el cual estaba integrado por un panel de seis expertos clínicos procedentes de diferentes regiones del país y por un grupo de profesionales con experiencia en la síntesis y el uso de evidencia en cáncer.
Se definieron 33 preguntas de interés clínico para el manejo quirúrgico integral, las cuales fueron consultadas y validadas en una conferencia nacional con especialistas clínicos de la Asociación. Las preguntas incluidas abordaron aspectos relacionados con la detección y el diagnóstico del cáncer epitelial de ovario, el manejo quirúrgico en estados tempranos y avanzados de la enfermedad, la combinación de la cirugía con otras modalidades como la quimioterapia, el seguimiento posterior al manejo primario, el diagnóstico y tratamiento de las recaídas posterior al manejo primario, y el manejo con intención paliativa.

Se llevó a cabo una revisión de literatura en dos etapas. En la primera se buscaron guías de práctica clínica basadas en evidencia que incluyeran las preguntas de interés. Las principales fuentes de información fueron las bases de datos de la Red Internacional de Guías (GIN, por su sigla en inglés) (12) y el repositorio de guías National Guideline Clearinghouse (NGC) de Estados Unidos (13). Se utilizaron términos de búsqueda relacionados con "cáncer de ovario", "neoplasias del ovario", "tumores del ovario" y "cáncer epitelial de ovario", no se realizaron restricciones por idioma, fuente o grupo desarrollador. A partir de las referencias encontradas, se seleccionaron guías de práctica clínica que hubieran sido publicadas entre enero de 2010 y diciembre de 2014. El cuerpo de guías encontradas se complementó con nuevas referencias citadas en los documentos hallados y mediante una búsqueda de literatura gris disponible en el buscador Google Académico. Se preguntó a expertos clínicos sobre la existencia de otras guías o consensos.

Del total de 25 documentos que fueron seleccionados y calificados con la herramienta AGREE II (14), se emplearon 13 guías de práctica clínica con sus tablas de evidencia como fuente de información (15-27). Se extrajeron las recomendaciones en una matriz diseñada para tal fin, que permitía registrar la evidencia fuente referenciada por cada desarrollador. Para garantizar la vigencia y exhaustividad de la evidencia, en una segunda etapa se llevaron a cabo revisiones de literatura dirigidas a identificar publi- 
caciones posteriores a la fecha de búsqueda de cada guía fuente, se seleccionaron revisiones sistemáticas, ensayos clínicos aleatorizados para las preguntas de intervención y, en algunos casos, estudios observacionales analíticos cuando no se encontraba mejor evidencia disponible. La evidencia fue recuperada de Medline, Embase y Cochrane Library.

Se construyeron tablas y resúmenes de evidencia para cada pregunta, y se formularon recomendaciones preliminares para cada una de ellas por parte de los expertos clínicos participantes; estas recomendaciones fueron revisadas y discutidas en una conferencia tipo panel de expertos organizada por la Asociación. El grado de fuerza de cada recomendación se estableció de acuerdo con el nivel de la evidencia de la cual proviene (tabla 2). Las recomendaciones finales y la evidencia que la soporta, así como las herramientas empleadas, pueden ser consultadas en detalle en la publicación completa de la guía (11).

El presente trabajo se desarrolló por expertos clínicos, de acuerdo con las consideraciones éticas para la investigación en humanos, garantizando la veracidad de los resultados y la idoneidad de los investigadores.

\section{RESULTADOS}

A continuación se presentan las recomendaciones de acuerdo con cada pregunta realizada. En la tabla 3 se resumen las recomendaciones clave.

\section{Detección temprana y diagnóstico del cáncer epitelial de ovario}

1. ¿Cuáles son los factores de riesgo asociados al cáncer epitelial de ovario (CEO)? (24, 28-34)

Es necesario conocer e identificar los factores de riesgo para cáncer de ovario en mujeres por encima de los 30 años, y en especial aquellas mayores de 50 años, para poder orientar su estudio en casos justificados.

Los factores de riesgo para desarrollar cáncer epitelial de ovario son:
- Nuliparidad

- Menarquia temprana

- Menopausia tardía

- Raza blanca

- Edad (mayores de 50 años)

- Ubicación geográfica (Norteamérica y norte de Europa)

- Historia familiar

- Historia personal de cáncer de seno

- Factores étnicos

No se ha comprobado que el uso de terapia de reemplazo hormonal, terapias de fertilidad, o el uso de talco sean factores de riesgo para el desarrollo de esta neoplasia.

Punto de buena práctica clínica

\section{2. ¿Cuáles son los signos y síntomas asociados con la presentación del CEO? (15, 35-50)}

Mujeres que asisten a la práctica médica general, que presenten uno o más síntomas de distensión abdominal con o sin dolor abdominal, dolor pélvico, sensación de llenura, dificultad para comer o síntomas urinarios, en los últimos tres meses, y que hayan requerido consultas repetidas, deben ser evaluadas para descartar un diagnóstico de cáncer de ovario, en especial si son mayores de 50 años.

A toda paciente con presencia de ascitis, masa abdominal o masa pélvica palpada en el examen médico o por la paciente, se le debe descartar un cáncer de ovario.

Punto de buena práctica clínica

\section{3. ¿Cuál es la utilidad diagnóstica de los índices de malignidad (Índice de riesgo de malignidad, ROMA) y los marcadores tumorales (CA125, HE4, ACE) para la aproximación diagnóstica de las pacientes con masa pélvica? (15, 17-19, 23, $24,26,27,51-56$ )}

Se recomienda utilizar los índices de riesgo de malignidad que combinan por lo menos dos pruebas para diferenciar entre una masa anexial benigna o maligna y así orientar su manejo. 
- Se recomienda utilizar el índice de riesgo de malignidad que tiene en cuenta los hallazgos del ultrasonido, el estado de menopausia y el valor del CA 125 con un punto de corte de 200 UI/ $\mathrm{mL}$ (ver Anexo 1).

- En menores de 40 años con masas complejas de ovario se deben solicitar otros marcadores (ACE, alfa FP, Beta HCG, LDH) para descartar tumores germinales o metastásicos.

- El algoritmo ROMA muestra buen rendimiento diagnóstico en mujeres posmenopáusicas para diferenciar entre una masa ovárica benigna y una maligna; sin embargo, su uso está limitado a aquellos centros en donde es posible medir el marcador sérico HE4.

Fuerza de la recomendación: moderada

\section{4. ¿Cuál es la utilidad diagnóstica del estudio} imagenológico con ecografía transvaginal o ecografía transabdominal para el diagnóstico de masa pélvica? $(15,18,23,24,26,27,51,53$, 57-59)

El examen primario de elección para confirmar una masa pélvica debe ser el ultrasonido transvaginal o, si no es posible, transabdominal.

- Si se encuentra disponible, es mejor realizarlo con técnica 3D para caracterizar las lesiones.

- No se recomienda utilizar resonancia magnética como primera opción para el diagnóstico de una masa pélvica, pues su sensibilidad y especificidad son similares a las del ultrasonido, pero su costo es mucho más elevado. Su uso solo está recomendado en caso de que no sea posible caracterizar la lesión por ultrasonido.

- Se recomienda el uso de la tomografía axial computarizada (TAC) para evaluar la presencia de enfermedad extrapélvica y caracterizarla.

Fuerza de la recomendación: moderada

\section{Consejería genética familiar}

5. ¿Qué pacientes deben ser remitidas a consejería genética familiar? $(26,28,51)$

Se recomienda remitir a consejería genética a aque- llas mujeres con antecedentes personales o familiares de parientes en primero o segundo grado que hayan presentado cáncer relacionado (seno, ovario o S. de Lynch). Especialmente si hay familiares que sean portadores de mutaciones BRCA 1 o 2 .

Punto de buena práctica clínica

\section{Generalidades del manejo quirúrgico inicial del cáncer epitelial de ovario (figura 1)}

6. ¿Qué debe hacer el cirujano no oncólogo (ginecólogo general, cirujano general) ante el hallazgo incidental de cáncer de ovario? $(24,60)$

Frente al hallazgo incidental de cáncer de ovario se recomienda:

- Registrar cuidadosamente todos los hallazgos en el protocolo intraoperatorio, tomar una biopsia y enviarla a patología.

- Remitir a la paciente a un equipo multidisciplinario especializado en cáncer ginecológico.

- Abstenerse de realizar procedimientos con cirugías citorreductoras hasta que la paciente tenga confirmación histológica y se planee una intervención adecuada para su diagnóstico histológico específico.

Fuerza de la recomendación: moderada

\section{7. ¿Cuáles son los criterios clínicos para la} remisión de la paciente con sospecha de CEO al ginecólogo oncólogo? (15, 18, 23, 25, 60-64)

Se recomienda remitir a ginecología oncológica a toda paciente con sospecha clínica de cáncer de ovario, bien sea por estudios imagenológicos o de marcadores tumorales, para manejo multidisciplinario.

- Pacientes con estudios de ultrasonido sospechosos de cáncer de ovario, CA 125 mayor de $200 \mathrm{UI} / \mathrm{mL}$ y ascitis deben ser remitidas a ginecología oncológica.

- Se recomienda el uso del índice de malignidad de Jacobs IRM 1 para clasificar los casos.

Fuerza de la recomendación: moderada 
8. ¿Cuál es la efectividad y seguridad de la salpingooforectomía bilateral (SOB) profiláctica en pacientes posmenopáusicas con y sin factores de riesgo? $(17,18,23,26,65-82)$

La salpingooforectomía bilateral está recomendada en mujeres con mutaciones BRCA1 y BRCA2, idealmente en edades entre 35 y 40 años y con paridad satisfecha. La edad puede modificarse de acuerdo con la edad más temprana de diagnóstico familiar de cáncer.

En mujeres posmenopáusicas sin factores de riesgo no hay información suficiente que permita recomendar o no su utilización. Se recomienda informar a las pacientes sobre el potencial riesgobeneficio de la salpingooforectomía bilateral de forma individual.

Fuerza de la recomendación: moderada

\section{9. ¿Cuál es el papel de la salpingectomía} profiláctica en paciente sin riesgo? (83-91)

Para mujeres con riesgo promedio de cáncer de ovario, quienes son llevadas a histerectomía por condiciones benignas, no hay estudios que permitan establecer una recomendación sobre el uso de la práctica de salpingectomía bilateral. Sin embargo, series de casos no han mostrado efectos adversos mayores al realizar este tipo de intervenciones.

Fuerza de la recomendación: débil

\section{0. ¿Cuáles son los pasos que se deben seguir} para la clasificación intraquirúrgica de las pacientes con cáncer de ovario? $(15,18,23,24$, 92-99)

Se recomienda realizar laparotomía de estadificación exhaustiva, la cual incluye laparotomía mediana para permitir la evaluación detallada del abdomen y la pelvis; histerectomía abdominal total; salpingooforectomía bilateral y omentectomía infracólica; biopsias de cualquier depósito peritoneal; biopsias aleatorias del peritoneo pélvico y abdominal; y la evaluación de ganglios linfáticos retroperitoneales mediante una resección ganglionar en caso de compromiso macroscópico o linfadenectomía pélvica y paraaórtica completa en caso de no encontrar lesión macroscópica, siempre que se logre citorreducción completa en la cavidad abdominal.

Procedimientos mínimamente invasivos pueden ser utilizados en sospecha de estadios tempranos en pacientes seleccionados, y deben ser realizados por ginecólogos oncólogos con experiencia.

Fuerza de la recomendación: moderada

\section{1. ¿Cuáles son los criterios prequirúrgicos} para definir entre citorreducción primaria y quimioterapia neoadyuvante? $(100,101)$

Se recomienda considerar la quimioterapia neoadyuvante en aquellas pacientes con compromiso severo de su estado general, o en aquellos estadios IIIC o IV, en los cuales no es posible garantizar una citorreducción completa.

- Aplicable en los casos que requieren una resección intestinal mayor de 1,5 m o múltiples resecciones intestinales.

- En caso de metástasis intrahepática o extraabdominal no resecable.

- En caso de tumor mayor de $2 \mathrm{~cm}$ alrededor de la arteria mesentérica superior o detrás de la porta hepática.

Punto de buena práctica clínica

\section{2. ¿Cuál debe ser el abordaje oncológico de la}

paciente con cáncer de ovario y cirugía previa incompleta? (15)

Para las pacientes con cirugía previa o estadificación incompleta se recomienda:

- En presuntos estadios IA o IB, grado I, completar cirugía y realizar estadificación quirúrgica.

- En presuntos estadios IA o IB, grados II o III, o células claras, completar cirugía y realizar estadificación quirúrgica.

- En presuntos estadios II, III, IV, realizar cirugía de reducción del tumor si hay sospecha de enfermedad residual potencialmente resecable. Si se sospecha enfermedad residual no resecable, 
realizar quimioterapia neoadyuvante y considerar la realización de cirugía de intervalo.

Nota: considere cirugía previa incompleta si existe: útero intacto, anexo intacto, omentum no removido, documentación de estatificación incompleta, enfermedad residual, potencialmente resecable, carcinoma invasivo oculto que se encontró en el momento de la cirugía de reducción del riesgo.

Punto de buena práctica clínica

\section{3. ¿Cuáles son las indicaciones y} contraindicaciones de la cirugía mínimamente invasiva en el manejo quirúrgico de cáncer de ovario? $(15,19,23-25,27,102-111)$

La laparoscopia es la vía preferible para la SOB profiláctica indicada en la mujer con riesgo hereditario de CEO. La laparoscopia puede ser la vía de acceso para pacientes con aparente CEO en estadios tempranos.

- La laparoscopia se puede utilizar como cirugía reestadificadora en pacientes con cirugía previa incompleta y aparente estadio temprano.

- En caso de sospecha de irresecabilidad por estudios imagenológicos, la laparoscopia puede utilizarse para la verificación de resecabilidad o irresecabilidad para el inicio de quimioterapia neoadyuvante. Deben describirse juiciosamente los hallazgos en la descripción. Se pueden utilizar índices validados en la literatura.

- La laparoscopia no muestra diferencia en evolución oncológica comparada con cirugía abierta en tumores infiltrantes en estadios tempranos o tumores borderline.

- La laparoscopia puede ser considerada en cirugía preservadora de la fertilidad.

- La laparoscopia debe ser discutida con el paciente y elegirse de acuerdo con preferencias de la paciente y del cirujano.

- En caso de decidirse la vía laparoscópica, esta siempre debe ser realizada por un ginecólogo oncólogo.

Fuerza de la recomendación: moderada

\section{Manejo del cáncer epitelial de ovario} en estadios tempranos (figura 1)

14. ¿Cuál es la efectividad del manejo

quirúrgico en estadios tempranos?

$(15,21,23-25,27,93,112-113)$

En estadios tempranos el manejo estándar recomendado es la cirugía, la cual incluye:

- Incisión mediana infraumbilical.

- Toma de muestra de ascitis o, en su ausencia, lavados peritoneales.

- Histerectomía abdominal + salpingooforectomía bilateral.

- Resección de lesiones peritoneales sospechosas o biopsias peritoneales aleatorias en caso de no evidencia de lesión macroscópica.

- Omentectomía infracólica.

- Linfadenectomía pélvica y paraaórtica.

En pacientes con deseo genésico se recomienda salpingooforectomía unilateral.

La vía laparoscópica puede considerarse para el diagnóstico y la estadificación, y ser parte del tratamiento en estadios tempranos; sin embargo, son necesarios estudios aleatorizados y controlados.

Fuerza de la recomendación: moderada

\section{5. ¿Cuáles son los sitios para la toma de} muestra de la citología intraoperatoria? (114)

Se debe tomar una muestra de líquido ascítico para citología. En ausencia de ascitis, se tomará muestra para citología con suero fisiológico de: fondo de saco anterior, fondo de saco posterior, goteras parietocólicas derecha e izquierda y citología de cúpulas diafragmáticas.

Punto de buena práctica clínica

16. ¿Cuál debe ser el esquema para la toma de biopsias peritoneales? (17, 23-25, 115-117)

La toma de biopsias peritoneales incluye:

- Visualizar y palpar todas las superficies peritoneales. 
- Resecar todas las adherencias sospechosas y no sospechosas.

- En ausencia de evidencia macroscópica de tumor, se deben tomar biopsias peritoneales aleatorias en fondo de saco anterior, fondo de saco posterior, goteras parietocólicas y cúpula diafragmática (raspado para citología).

Fuerza de la recomendación: débil

17. ¿Cuál es el papel de la apendicectomía

en el manejo de la paciente con cáncer de ovario? (23-25, 118-122)

Se debe realizar apendicectomía en caso de evidencia macroscópica de compromiso tumoral. También se recomienda este procedimiento en tumores mucinosos y seudomixoma peritoneal.

Fuerza de la recomendación: débil

18. ¿Cuál es la efectividad y seguridad de la linfadenectomía radical frente al muestreo ganglionar en estadios tempranos?

$(15,25,94,96)$

Se recomienda realizar linfadenectomía pélvica y paraaórtica en las pacientes con cáncer epitelial de ovario en estadios tempranos.

Fuerza de la recomendación: moderada

19. ¿Cuáles son las indicaciones y rol de la biopsia por congelación durante el abordaje quirúrgico? (18, 23, 27, 96, 112, 123-125)

Se recomienda el uso de la biopsia por congelación para confirmar enfermedad maligna y desarrollar un algoritmo de manejo individual.

- Se deben considerar las limitaciones de la misma en casos de tumores con bajo riesgo de malignidad.

- Las pacientes que deseen mantener potencialmente la fertilidad deben tener una evaluación intraoperatoria con biopsia por congelación.

- En cirugía de preservación de la fertilidad se puede realizar (si es técnicamente factible) si los resultados de la biopsia por congelación son positivos para tumor maligno de células germinales, cáncer epitelial de ovario estadio I o tumores borderline.

- Se debe evitar hacer procedimientos radicales sin biopsia por congelación en casos en los que exista duda.

Fuerza de la recomendación: moderada

20. ¿Cuál es la indicación de quimioterapia

posoperatoria en estadios tempranos?

$(15,23-25,95,123,126)$

Se recomienda el uso de adyuvancia en estadios IA (G3), IB (G2, G3), IC y IIA, en tumores de células claras en cualquier grado o estadio, durante tres a seis (6) ciclos con quimioterapia complementaria.

Fuerza de la recomendación: moderada

\section{Condiciones especiales (figura 2)}

21. ¿Cuál es el manejo quirúrgico de la paciente con diagnóstico de CEO borderline? (22, 23, 27, 127-133)

En mujeres con cirugía completa la paciente debe ser llevada a observación. En caso de cirugía incompleta, evaluar el deseo de fertilidad.

En mujeres menores de 40 años debe considerarse cirugía conservadora de fertilidad si la paciente lo desea.

- En caso de cirugía conservadora de fertilidad:

- SOU (para tumor unilateral). Considerar cistectomía en caso de compromiso bilateral.

- Exploración de cavidad (resección de implantes).

- En caso de no preservación de fertilidad

- Histerectomía abdominal.

- SOB.

- Exploración cavidad (resección de implantes).

- Se debe realizar citorreducción completa en caso de recaídas.

La laparoscopia es una opción posible para el manejo de los tumores borderline.

Fuerza de la recomendación: débil 
22. ¿Cuáles son las condiciones para realizar cirugía conservadora de fertilidad en paciente con CEO en estadios iniciales? $(18,23-25,27,128$, 130, 134-141)

La cirugía conservadora de fertilidad puede tenerse en cuenta en mujeres menores de 40 años que deseen paridad, con estadios IA (grados Iy II) y IC (grado 1).

- La paciente debe recibir información completa del procedimiento y posibles desenlaces.

- Debe incluirse estadificación completa de la enfermedad como parte del procedimiento.

- En casos seleccionados se sugiere utilizar la vía laparoscópica.

- Debe considerarse completar cirugía cuando esté satisfecho el deseo de paridad.

Fuerza de la recomendación: moderada

\section{Manejo del cáncer epitelial de ovario en estadios avanzados (figura 1)}

23. ¿Cuál es la efectividad y seguridad de la cirugía ultrarradical en el manejo de la paciente con CEO avanzado? (9, 10, 15, 18-20, 23-25, 94, 142-149)

Se recomienda la cirugía como manejo estándar inicial de la paciente con cáncer epitelial de ovario, con el objetivo de clasificar la enfermedad y lograr una citorreducción completa.

La cirugía radical debe incluir: histerectomía abdominal, salpingooforectomía bilateral, omentectomía, linfadenectomía pélvica y paraaórtica, resección de superficies peritoneales, resecciones intestinales, resecciones hepáticas, cola de páncreas y esplenectomía. Estas cinco últimas solo en caso de evidencia de tumor macroscópico de estos órganos.

Fuerza de la recomendación: moderada

\section{4. ¿Cuáles son las estrategias para lograr una} citorreducción completa u óptima en pacientes con CEO avanzado? (9, 18, 23-25, 142, 143, 150, 151)

Se recomienda la citorreducción completa como objetivo quirúrgico, para lo cual se prefiere el abordaje ultrarradical en pacientes con CEO avanzado.
Se recomienda acceder al abdomen superior como parte del abordaje ultrarradical, para lo cual es indispensable desarrollar equipos de trabajo multidisciplinarios.

Fuerza de la recomendación: moderada

\section{5. ¿Cuál es la efectividad y seguridad de la linfadenectomía en la paciente con CEO avanzado? (Opinión de expertos)}

Se recomienda hacer linfadenectomía pélvica y paraaórtica en pacientes con cáncer epitelial de ovario en estadio avanzado solamente cuando se logre la citorreducción completa o exista evidencia de compromiso ganglionar (imágenes o palpación).

Fuerza de la recomendación: fuerte

\section{6. ¿Cuáles son las indicaciones y el alcance de la citorreducción secundaria en pacientes con CEO? (18, 24, 25, 152-158)}

Se recomienda la citorreducción secundaria en pacientes sensibles al platino que recaen en forma loco-regional seis meses o más posteriores al tratamiento primario.

El objetivo debe ser la citorreducción completa. Fuerza de la recomendación: moderada

27. ¿Cuál es el momento adecuado durante la quimioterapia neoadyuvante para realizar Ia cirugía de intervalo? $(9,15,18,23-25,101$, 159-162)

Se sugiere la neoadyuvancia como alternativa terapéutica en el grupo de pacientes de alta comorbilidad, estadios IIIC/IV, o en aquellos que cumplan los criterios de Leuven para irresecabilidad.

No existe consenso o evidencia acerca del número de ciclos de quimioterapia previos a la cirugía de intervalo.

Al terminar el 3 o 4 ciclo de quimioterapia se debe realizar evaluación clínica e imagenológica y CA125 si este ha estado elevado previamente, con el fin de decidir la cirugía de intervalo.

Fuerza de la recomendación: fuerte 
28. ¿Cuáles son las indicaciones de la quimioterapia intraperitoneal en estadios avanzados? (18-20, 25, 163-169)

La quimioterapia intraperitoneal (IP) debe considerarse de forma individual, en casos de cáncer epitelial de ovario avanzados (estadio III o más), en consenso con oncología clínica, el paciente y su familia, en pacientes con citorreducción óptima atendidas en centros especializados con capacidad de manejo integral.

Se recomienda limitar el uso de quimioterapia intraperitoneal hipertérmica (HIPEC) en el abordaje primario o en la recaída del CEO a pacientes en estados avanzados atendidas en centros especializados exclusivamente en el marco de estudios clínicos.

Fuerza de la recomendación: fuerte

\section{Seguimiento y manejo de las recaídas}

29. ¿Cuál es el esquema adecuado para el seguimiento en las pacientes tratadas por CEO? $(18,23-25,170,171)$

En cada visita de seguimiento debe realizarse una historia clínica y un examen físico completos, incluyendo el examen pélvico. Si no hay síntomas sugestivos de recaída (similares a los de un tumor primario), no hay obligatoriedad de tomar ningún examen paraclínico. De acuerdo con lo hallado en la entrevista o el examen físico puede, en cada caso, ser necesario ordenar niveles de CA125 u otros marcadores tumorales cuando aplique (como antígeno carcinoembrionario), particularmente si estuvieron elevados anteriormente, y en aquellos casos en los que se considere que con una detección temprana es posible una citorreducción secundaria benéfica para la paciente; así mismo, se debe considerar tomar imágenes diagnósticas (rayos X, ecografía, resonancia magnética, tomografía axial, PET-CT).

En caso sugestivo de recaída se recomienda valorar a la paciente en junta multidisciplinaria. Las visitas de seguimiento tras el tratamiento deben ser realizadas cada 3-4 meses los primeros dos años, cada 6 meses entre el tercer y quinto año y anualmente a partir del quinto año.

Punto de buena práctica clínica
En los casos en los cuales sea realizada quimioterapia adyuvante, el control debe realizarse también por el ginecólogo oncólogo con periodicidad bimestral mientras se está realizando su administración.

Fuerza de la recomendación: moderada

\section{0. ¿Cómo se define la recaída posterior al tratamiento en pacientes con CEO? $(23,171)$}

La recaída posterior al tratamiento en pacientes con CEO hace referencia a la aparición de la enfermedad después del tratamiento primario. Esta puede ser clasificada como clínica o serológica.

- Recaída clínica. Aparición de síntomas sugestivos de la enfermedad (similares a los del tumor primario) o atribuidos a localizaciones secundarias, con evidencia de tumor en el examen clínico o en las imágenes que se practiquen.

- Recaída serológica

- Pacientes con elevación del CA125 antes del tratamiento en quienes se haya normalizado: elevación igual o superior a $70 \mathrm{UI} / \mathrm{mL}$ en dos muestras tomadas con al menos una semana de diferencia ${ }^{1}$ (dos veces el valor superior normal).

- Pacientes con elevación del CA125 antes del tratamiento en quienes nunca se normalizó: elevación de dos veces el nadir (valor mínimo alcanzado) en dos muestras tomadas con al menos una semana de diferencia.

- Pacientes con valores normales de CA125 antes del tratamiento: elevación igual o superior a $70 \mathrm{UI} / \mathrm{mL}$ en dos muestras tomadas con al menos una semana de diferencia.

Punto de buena práctica clínica

\section{1. ¿Cómo debe ser el estudio y manejo de la recaída exclusivamente serológica en pacientes con CEO? (18, 23, 171-179)}

En casos de recaída serológica deben tomarse estudios imagenológicos conducentes a identificar la lo-

1 Se debe tener presente que la vida útil del CA125 es de máximo dos semanas, por tanto, la segunda prueba debe tomarse en este periodo. 
calización y extensión de la enfermedad recurrente con el objetivo de planear la conducta subsecuente. Resultan de utilidad la TAC abdominopélvica con contraste, y de tórax, la resonancia magnética y la tomografía por emisión de positrones en combinación con la TAC (PET-CT). En todos los casos se recomienda definir en una junta médica multidisciplinaria la conducta que se debe seguir.

En caso de documentarse una recaída exclusivamente serológica, sin evidencia de representación clínica ni imagenológica, en pacientes con cáncer de ovario, la paciente debe ser evaluada por una junta médica que incluya las especialidades de ginecología oncológica y oncología clínica, y que formule una recomendación ante las circunstancias individuales. Las posibilidades de manejo de esta condición pueden seguir diferentes recomendaciones tales como postergar el inicio de tratamiento hasta que existan manifestaciones clínicas (síntomas) o considerar otras opciones como el inicio de tratamiento con quimioterapia, terapia hormonal, o la incorporación de la paciente en ensayos clínicos.

Punto de buena práctica clínica

\section{Manejo paliativo del cáncer epitelial de ovario}

32. ¿Cuáles son los criterios para declarar una paciente como no susceptible de manejo con intención curativa y remitir a cuidado paliativo? $(23,180)$

En las pacientes con persistencia, progresión de la enfermedad o toxicidad limitante, debe decidirse si serán sometidas a otras líneas de quimioterapia o se reorienta la intención del tratamiento hacia el control de los síntomas y cuidados de soporte. Estas pacientes también podrían incluirse en ensayos clínicos donde estén disponibles.

Punto de buena práctica clínica
33. ¿Cómo debe ser el manejo paliativo de la obstrucción intestinal en la paciente con CEO avanzado? (18, 180-186)

Los procedimientos de intención paliativa en las pacientes con cáncer de ovario deben realizarse para el tratamiento de situaciones específicas y cuando se considere que ofrecerán un beneficio clínico significativo. La cirugía para obstrucción intestinal debe ser realizada solo en casos de obstrucción mecánica y no de manera preventiva. Debe evitarse realizar cirugía para obstrucción intestinal ante situaciones como enfermedad metastásica difusa demostrada en cirugía previa, compromiso intestinal proximal, enfermedad metastásica masiva palpable, ascitis severa rápidamente recurrente o la oposición de la paciente. Debe considerarse también evitarla en casos de compromiso extraabdominal extenso y en pacientes con mal estado funcional o nutricional.

Fuerza de la recomendación: fuerte

\section{ACTUALIZACIÓN DE LAS RECOMENDACIONES}

El grupo desarrollador de la guía recomienda hacer la actualización de las recomendaciones en un periodo no mayor a cuatro años a partir de la fecha de publicación del presente documento, o cuando exista nueva evidencia que pueda cambiar las recomendaciones formuladas.

\section{DECLARACIÓN DE CONFLICTOS DE INTERÉS Y FINANCIACIÓN}

En las diferentes actividades, los participantes en el desarrollo de la guía declararon no tener algún conflicto de interés que pudiera interferir en las actividades y la formulación de recomendaciones. En todo el proceso los integrantes del grupo desarrollador y los participantes en el proyecto realizaron la declaración escrita y firmada de potenciales conflictos de interés, los cuales han sido analizados y se encuentran a disposición de los interesados. 
Los recursos para el desarrollo de la guía y del presente artículo provienen de una subvención para investigación y soporte logístico de Productos Roche S.A. La entidad financiadora garantizó total independencia de las acciones y opiniones de los expertos del panel, especialistas participantes y grupo de apoyo metodológico.

Nota: este artículo hace parte de la versión completa de la "Guía de práctica clínica (GPC) para el manejo quirúrgico del cáncer epitelial de ovario en Colombia. Bogotá" (11). Este documento es publicado con autorización de la Asociación Colombiana de Ginecólogos Oncólogos y el Instituto para la Evaluación de la Calidad y Atención en Salud (IECAS).

\section{"REPRESENTANTES DEL GRUPO DESARROLLADOR DE LA GUIIA}

Jesús Antonio Acosta-Peñaloza, MD. Ginecólogo oncólogo, Asociación Colombiana de Ginecólogos Oncólogos; Instituto Nacional de Cancerología ESE, Bogotá (Colombia). jesacosta3@gmail.com

Mauricio González-Castañeda, MD. Ginecólogo oncólogo, Asociación Colombiana de Ginecólogos Oncólogos, Instituto Nacional de Cancerología ESE, Bogotá (Colombia).

Robinson Fernández-Mercado, MD. Ginecólogo oncólogo, Asociación Colombiana de Ginecólogos Oncólogos, Bogotá (Colombia); Misión Médica Ltda., Barranquilla (Colombia).

Heydi Marina Ramírez-Salazar, MD. Ginecóloga oncóloga, Asociación Colombiana de Ginecólogos Oncólogos, Bogotá (Colombia); Fundación Clínica Valle de Lili, Cali (Colombia).

Jaime Fernando Ruiz-Povea, MD. Ginecólogo oncólogo, Asociación Colombiana de Ginecólogos Oncólogos; Oncólogos de Occidente, Manizales (Colombia).

Daniel Sanabria-Serrano, MD. Ginecólogo oncólogo, Asociación Colombiana de Ginecólogos Oncólogos; Fundación Santa Fe de Bogotá, Bogotá (Colombia).

Carolina Sandoval-Salinas. Bacterióloga; Especialista en Epidemiología general, Instituto para la Evaluación de la Calidad y la Atención en Salud (IECAS), Bogotá (Colombia).

Giancarlo Buitrago-Gutiérrez, MD, MSc. Médico cirujano; MSc en Epidemiología clínica; MSc en Economía, Instituto para la Evaluación de la Calidad y la Atención en Salud (IECAS), Bogotá (Colombia).

Juan Sebastián Castillo-Londoño, MD, MSc. Médico cirujano; MSc en Epidemiología clínica; MSc en Salud pública. Instituto para la Evaluación de la Calidad y la Atención en Salud (IECAS), Bogotá (Colombia).

\section{REVISIÓN DE LA GUIIA}

Grupo Clínica Ginecología, Instituto Nacional de Cancerología.

\section{REFERENCIAS}

1. Wei W, Dizon D, Vathipadiekal V, Birrer MJ. Ovarian Cancer: genomic analysis. Annals of Oncology. 2013;24(suppl10):S7-S10.

2. Ulusoy S, AKbayir O, Nomanoglu C, Ulusoy N, Odabas E, Gulkilik A. The risk of malignancy index in discrimination of adnexal masses. Int J Gynaecol Obstet. 2007;96:186-91.

3. Instituto Nacional de Cancerología. Estadísticas | Instituto Nacional de Cancerología [Internet]. 2013 [cited 2015 Sept 9]. Disponible en: http://www.cancer. gov.co/content/estadisticas

4. Schorge JO, Eisenhauer EE, Chi DS. Current surgical management of ovarian cancer. Hematol Oncol Clin North Am. 2012;26:93-109.

5. Tavassoli FA, Devilee P. World Health Organization Classification of Tumors. Pathology and Genetics of Tumors of the Breast and Female Genital Organs. Lyon: IARC Press; 2003.

6. Colombo N, VanGorp T, Parma G, Amant F, Gatta G, Sessa, et al. Ovarian cancer. Crit Rev Oncol Hematol. 2006;60:159-79.

7. Rufford BD, Jacobs IJ, Menon U. Feasibility of screening for ovarian cancer using symptoms as selection criteria. BJOG. 2007;114:59-64.

8. Harter P, Muallem ZM, Buhrmann C, Lorenz D, Kaub C, Hils R, et al. Impact of a structured quality management program on surgical outcome in primary advanced ovarian cancer. Gynecol Oncol. 2011; 121:615-9.

9. Chi DS, Eisenhauer EL, Zivanovic O, Sonoda Y, AbuRustum NR, Levine DA, et al. Improved progressionfree and overall survival in advanced ovarian cancer as a result of a change in surgical paradigm. Gynecol Oncol. 2009;114:26-31.

10. Vergote I, Tropé CG, Amant F, Kristensen GB, Ehlen T, Johnson N, et al. Neoadjuvant chemotherapy or primary surgery in stage IIIC or IV ovarian cancer. N Eng J Med. 2010;363:943-53.

11. Asociación Colombiana de Ginecólogos Oncólogos. Instituto para la Evaluación de la Calidad y Atención en Salud (IECAS). Guía de práctica clínica (GPC) para el 
manejo quirúrgico del cáncer epitelial de ovario en Colombia. Bogotá. En proceso de publicación electrónica.

12. Guidelines International Network. G-I-N Guidelines International Network [Internet]. 2013 [cited 2015 Sept 8]. Disponible en: http://www.g-i-n.net/

13. Agency for Healthcare Research and Quality. National Guideline Clearinghouse [Internet] [visitado 2015 Sept 9]. Disponible en: http://www.guideline.gov/

14. Brouwers MC, Kho ME, Browman GP, Burgers JS, Cluzeau F, Feder G, et al. AGREE Next Steps Consortium; AGREE II: advancing guideline development, reporting and evaluation in health care. CMAJ. 2010;182:E839-42.

15. National Institute of Clinical Excellence NICE. Ovarian cancer: the recognition and initial management of ovarian cancer. Wales; 2011.

16. Moyer VA, Services USP, Force T. Clinical Guideline Screening for Ovarian Cancer: US Preventive Services Task Force OF. 2012;157(12).

17. National Breast and Ovarian Cancer Centre. Recommendations for management of women at high risk of ovarian cancer; 2011.

18. Scottish Intercollegiate Guidelines Network (SIGN). Management of epithelial ovarian cancer. Edinburgh; 2013 [visitado 2015 Sept 9]. Disponible en: http:// www.sign.ac.uk

19. Royal College of Obstetricians and Gynaecologist. Management of Suspected Ovarian Masses in Premenopausal Women. Green-top Guideline No. 62 RCOG/BSGE; 2011.

20. National Institute of Clinical Excellence NICE. Ovarian Cancer : Evidence Update January 2013. A summary of selected new evidence relevant to NICE clinical guideline 122, “The recognition and initial management of ovarian cancer" (2011) Evidence. Manchester; 2013.

21. National Institute of Clinical Excellence NICE. Quality standard for ovarian cancer. Manchester; 2012.

22. National Institute of Clinical Excellence NICE. Ovarian cancer overview. NICE Pathways [Internet]. Manchester; 2014 [visitado 2015 Sept. 9]. Disponible en: http:// pathways.nice.org.uk/pathways/ovarian-cancer
23. National Comprehensive Cancer Network. Ovarian Cancer including Fallopian Tube Cancer and Primary Peritoneal Cancer. Version 3.2014; 2014.

24. Ministerio de Salud. Guía clínica AUGE Ovario Epitelial. Santiago de Chile; 2013.

25. Alberta Provincial Gynecologic Oncology Tumour Team. Clinical practice guideline Gyne-005, version 3. Epithelial ovarian, fallopian tube, and primary peritoneal cancer; 2013.

26. Alberta Provincial Gynecologic Oncology Tumour. Clinical practice guideline Br-011, version 2. Risk reduction and surveillance strategies for individuals at high genetic risk for breast and ovarian cancer; 2011.

27. Dodge J, Covens A, Lacchetti C, Elit L, Le T, Fung MFK. Management of a Suspicious Adnexal Mass. Toronto; 2011.

28. Jelovac D, Armstrong DK. Recent Progress in the Diagnosis and Treatment of Ovarian Cancer. CA Cancer J Clin. 2011;61:183-203.

29. Holschneider CH, Berek JS. Ovarian Cancer : Epidemiology, Biology, and Prognostic Factors. Semin Surg Oncol. 2000;19:3-10.

30. Pearce CL, Templeman C, Rossing MA, Lee A, Near AM, Webb PM, et al. Association between endometriosis and risk of histological subtypes of ovarian cancer: a pooled analysis of case-control studies. Lancet Oncol. 2012;13:385-94.

31. Faber MT, Kjær SK, Dehlendorff C, Chang-Claude J, Andersen KK, Høgdall E, et al. Cigarette smoking and risk of ovarian cancer: a pooled analysis of 21 case-control studies. Cancer Causes Control. 2013;24:989-1004.

32. Mørch LS, Andreasen AH, Krüger-kjær S. Hormone Therapy and Ovarian Cancer. JAMA. 2009;302:298-305.

33. Mørch LS, Løkkegaard E, Andreasen AH, Kjaer SK, Lidegaard O. Hormone therapy and different ovarian cancers: a national cohort study. Am J Epidemiol. 2012;175:1234-42.

34. van Leeuwen FE, Klip H, Mooij TM, van de Swaluw AM, Lambalk CB, Kortman M, et al. Risk of borderline and invasive ovarian tumors after ovarian stimulation for in vitro fertilization in a large Dutch cohort. Hum Reprod. 2011;26:3456-65. 
35. Flam F, Einhorn N, Sjövall K. Symptomatology of ovarian cancer. Eur J Obstet Gynecol Reprod Biol. 1988;27:53-7.

36. Olson SH, Mignone L, Nakraseive C, Caputo TA, Barakat RR, Harlap S. Symptoms of Ovarian Cancer. Obstet Gynecol. 2001;98:212-7.

37. Goff BA, Mandel LS, Drescher CW, Urban N, Gough S, Schurman KM, et al. Development of an ovarian cancer symptom index: possibilities for earlier detection. Cancer. 2007;109:221-7.

38. Buys SS, Patridge E, Black A, Johnson CC, Lamerato $\mathrm{L}$, Isaacs C, et al. Effect of screening on ovarian cancer mortality: The Prostate, Lung, Colorectal and Ovarian (PLCO) Cancer Screening Randomized Controlled Trial. JAMA. 2011;305:2295-303.

39. Andersen MR, Goff BA, Lowe KA, Scholler N, Bergan L, Drescher CW, et al. Use of a Symptom Index, CA125, and HE4 to predict ovarian cancer. Gynecol Oncol. 2010;116:378-83.

40. Bankhead CR, Kehoe ST, Austoker J. Symptoms associated with diagnosis of ovarian cancer: a systematic review. BJOG. 2005;112:857-65.

41. Bankhead CR, Collins C, Stokes-Lampard H, Rose P, Wilson S, Clements A, et al. Identifying symptoms of ovarian cancer: a qualitative and quantitative study. BJOG. 2008;115:1008-14.

42. Friedman GD, Skilling JS, Udaltsova NV, Smith LH. Early symptoms of ovarian cancer: a case-control study without recall bias. Fam Pract. 2005;22:548-53.

43. Goff BA, Mandel LS, Melancon CH, Muntz HG. Frequency of symptoms of ovarian cancer in women presenting to primary care clinics. JAMA. 2004;291:2705-12.

44. Hamilton W, Peters TJ, Bankhead C, Sharp D. Risk of ovarian cancer in women with symptoms in primary care: population based case-control study. BMJ. 2009;339:b2998.

45. Kim MK, Kim K, Kim SM, Kim JW, Park NH, Song YS, et al. A hospital-based case-control study of identifying ovarian cancer using symptom index. J Gynecol Oncol. 2009;20:238-42.

46. Lurie G, Thompson PJ, McDuffie KE, Carney ME, Goodman MT. Prediagnostic symptoms of ovarian carcinoma: a case-control study. Gynecol Oncol. 2009; 114:231-6.

47. Olsen CM, Cnossen J, Green AC, Webb PM. Comparison of symptoms and presentation of women with benign, low malignant potential and invasive ovarian tumors. Eur J Gynaecol Oncol. 2007;28:376-80.

48. Rossing MA, Wicklund KG, Cushing-Haugen KL, Weiss NS. Predictive value of symptoms for early detection of ovarian cancer. J Natl Cancer Inst. 2010; 102:222-9.

49. Vine MF, Ness RB, Calingaert B, Schildkraut JM, Berchuck A. Types and duration of symptoms prior to diagnosis of invasive or borderline ovarian tumor. Gynecol Oncol. 2001;83:466-71.

50. Webb PM, Purdie DM, Grover S, Jordan S, Dick ML, Green AC. Symptoms and diagnosis of borderline, early and advanced epithelial ovarian cancer. Gynecol Oncol. 2004;92:232-9.

51. Lancaster JM, Powell CB, Chen L, Richardson DL. Society of Gynecologic Oncology statement on risk assessment for inherited gynecologic cancer predispositions. Gynecol Oncol. 2015;136:3-7.

52. Dodge JE, Covens AL, Lacchetti C, Elit LM, Le T, Devries-Aboud M, et al. Preoperative identification of a suspicious adnexal mass: a systematic review and meta-analysis. Gynecol Oncol. 2012;126:157-66.

53. Geomini P, Kruitwagen R, Bremer G, Cnossen J, Mol B. The Accuracy of Risk Scores in Predicting Ovarian Malignancy. A sistematic review. Obstet Gynecol. 2009;113(2):384-94.

54. Kaijser J, Sayasneh A, van Hoorde K, Ghaem-Maghami S, Bourne T, Timmerman D, et al. Presurgical diagnosis of adnexal tumors using mathematical models and scoring systems: a systematic review and meta-analysis. Hum Reprod Update. 2014;20:449-62.

55. Myers ER, Bastian LA, Havrilesky LJ, Kulasingam SL, Terplan MS, Cline KE, et al. Management of Adnexal Mass. Evid Rep Technol Assess. 2006:1-145.

56. Testa A, Kaijser J, Wynants L, Fischerova D, van Holsbeke C, Franchi D, et al. Strategies to diagnose ovarian cancer: new evidence from phase 3 of the multicentre international IOTA study. Br J Cancer. 2014;111:680-8. 
57. Wang J, Gao J, Yao H, Wu Z, Wang M, Qi J. Diagnostic accuracy of serum HE4, CA125 and ROMA in patients with ovarian cancer: a meta-analysis. Tumor Biol. 2014;35:6127-38.

58. Medeiros LR, Rosa DD, da Rosa MI, Bozzetti MC. Accuracy of CA 125 in the diagnosis of ovarian tumors: a quantitative systematic review. Eur J Obstet Gynecol Reprod Biol. 2009;142:99-105.

59. Liu J, Xu Y, Wang J. Ultrasonography, computed tomography and magnetic resonance imaging for diagnosis of ovarian carcinoma. Eur J Radiol [Internet]. 2007;62:328-34.

60. Vernooij F, Heintz P, Witteveen E, van der Graaf Y. The outcomes of ovarian cancer treatment are better when provided by gynecologic oncologists and in specialized hospitals: a systematic review. Gynecol Oncol. 2007;105:801-12.

61. Du Bois A, Rochon J, Pfisterer J, Hoskins WJ. Variations in institutional infrastructure, physician specialization and experience, and outcome in ovarian cancer: a systematic review. Gynecol Oncol. 2009;112:422-36.

62. Giede KC, Kieser K, Dodge J, Rosen B. Who should operate on patients with ovarian cancer? An evidencebased review. Gynecol Oncol. 2005;99:447-61.

63. American College of Obstetricians and Gynecologists. ACOG Committee Opinion: number 280, December 2002. The role of the generalist obstetrician-gynecologist in the early detection of ovarian cancer. Obstet Gynecol. 2002;100:1413-16.

64. Im SS, Gordon AN, Buttin BM, Iii CAL, Gostout BS, Shah C, et al. Validation of Referral Guidelines for Women with Pelvic Masses. Am Coll Obstet Gynecol. 2005;105:35-41.

65. Finch A, Evans G, Narod SA. BRCA carriers, prophylactic salpingo-oophorectomy and menopause: clinical management considerations and recommendations. Womens Health (Lond Engl). 2012;8:543-55.

66. Finch A, Beiner M, Lubinski J, Lynch HT, Moller P, Rosen B, et al. Salpingo-oophorectomy and the risk of ovarian, fallopian tube, and peritoneal cancers in women with a BRCA1 or BRCA2 Mutation. JAMA. 2006;296:185-92.
67. Domchek SM, Friebel TM, Singer CF, Evans DG, Lynch HT, Isaacs C, et al. Association of risk-reducing surgery in BRCA1 or BRCA2 mutation carriers with cancer risk and mortality. JAMA. 2010;304:967-75.

68. Horsman D, Wilson BJ, Avard D, Meschino WS, Kim Sing C, Plante M, et al. Clinical management recommendations for surveillance and risk-reduction strategies for hereditary breast and ovarian cancer among individuals carrying a deleterious BRCA1 or BRCA2 mutation. J Obstet Gynaecol Can. 2007;29:45-60.

69. Domchek SM, Weber BL. Clinical management of BRCA1 and BRCA2 mutation carriers. Oncogene. 2006;25:5825-31.

70. Kurian AW, Sigal BM, Plevritis SK. Survival analysis of cancer risk reduction strategies for BRCA1/2 mutation carriers. J Clin Oncol. 2010;28:222-31.

71. Armstrong K, Schwartz JS, Randall T, Rubin SC, Weber B. Hormone replacement therapy and life expectancy after prophylactic oophorectomy in women with BRCA1/2 mutations: a decision analysis. J Clin Oncol. 2004;22:1045-54.

72. Rebbeck TR, Kauff ND, Domchek SM. Meta-analysis of risk reduction estimates associated with risk-reducing salpingo-oophorectomy in BRCA1 or BRCA2 mutation carriers. J Natl Cancer Inst. 2009;101:80-7.

73. Daly MB, Pilarski R, Axilbund JE, Buys SS, Crawford B, Friedman S, et al. Genetic/familial high-risk assessment: breast and ovarian, version 1.2014. J Natl Compr Canc Netw. 2014;12:1326-38.

74. Movahedi S, Baker PM, Gilks B, Soslow RA, Oliva E. Protocol for the Examination of Specimens from Patients with Carcinoma of the Ovary. College of American Pathologists (CAP); 2009 [visitado 2015 Sept 9]. Disponible en: http://www.cap.org/apps/ docs/committees/cancer/cancer_protocols/2009/Ov y_09protocol.pdf

75. Finch A, Shaw P, Rosen B, Murphy J, Narod SA, Colgan TJ. Clinical and pathologic findings of prophylactic salpingo-oophorectomies in 159 BRCA1 and BRCA2 carriers. Gynecol Oncol. 2006;100:58-64.

76. Rebbeck TR, Lynch HT, Neuhausen SL, Narod SA, Van't Veer L, Garber JE, et al. Prophylactic oophorec- 
tomy in carriers of BRCA1 or BRCA2 mutations. $\mathrm{N}$ Engl J Med. 2002;346:1616-22.

77. Kauff ND, Satagopan JM, Robson ME, Scheuer L, Hensley M, Hudis CA, et al. Risk-reducing salpingooophorectomy in women with a BRCA1 or BRCA2 mutation. N Engl J Med. 2002;346:1609-15.

78. Rebbeck TR, Levin AM, Eisen A, Snyder C, Watson P, Cannon-Albright L, et al. Breast cancer risk after bilateral prophylactic oophorectomy in BRCA1 mutation carriers. J Natl Cancer Inst. 1999;91:1475-9.

79. Eisen A, Lubinski J, Klijn J, Moller P, Lynch HT, Offit $\mathrm{K}$, et al. Breast cancer risk following bilateral oophorectomy in BRCA1 and BRCA2 mutation carriers: an international case-control study. J Clin Oncol. 2005;23:7491-6.

80. Berek JS, Chalas E, Edelson M, Moore DH, Burke WM, Cliby WA, et al. Prophylactic and risk-reducing bilateral salpingo-oophorectomy: recommendations based on risk of ovarian cancer. Obstet Gynecol. 2010; 116:733-43.

81. Finch AP, Lubinski J, Moller P, Singer CF, Karlan B, Senter L, et al. Impact of oophorectomy on cancer incidence and mortality in women with a BRCA1 or BRCA2 mutation. J Clin Oncol. 2014;32:1547-53.

82. Marchetti C, De Felice F, Palaia I, Perniola G, Musella A, Musio D, et al. Risk-reducing salpingo-oophorectomy: a meta-analysis on impact on ovarian cancer risk and all cause mortality in BRCA 1 and BRCA 2 mutation carriers. BMC Womens Health. 2014;14:150.

83. Schenberg T, Mitchell G. Prophylactic bilateral salpingectomy as a prevention strategy in women at high-risk of ovarian cancer: a mini-review. Front Oncol. 2014;4:21.

84. Morelli M, Venturella R, Mocciaro R, Di Cello A, Rania E, Lico D, et al. Pro-phylactic salpingectomy in premenopausal low-risk women for ovarian cancer: primum non nocere. Gynecol Oncol. 2013;129:448-51.

85. Finch A, Narod SA. Quality of life and health status after prophylactic sal-pingo-oophorectomy in women who carrya BRCA mutation: A review. Maturitas.2011;70:261-5.

86. Finch A, Metcalfe KA, Chiang J, Elit L, McLaughlin J, Springate C, et al. The impact of prophylactic salpingo-oophorectomy on quality of life and psy- chological distress in women with a BRCA mutation. Psychooncology.2013; 22:212-9.

87. Pezaro C, James P, McKinley J, Shanahan M, Young MA, Mitchell G. The consequences of risk reducing salpingo-oophorectomy: the case for a coordinated approach to long-term follow up post surgical menopause. Fam Cancer.2012;11:403-10.

88. Antoniou AC, Rookus M, Andrieu N, Brohet R, Chang-Claude J, Peock S, et al. Reproductive and hormonal factors, and ovarian cáncer risk for BRCA1 and BRCA2 mutation carriers: results from the International BRCA1/2 Carrier Cohort Study. Cancer Epidemiol Biomarkers Prev.2009;18:601-10.

89. Rocca WA, Grossardt BR, de Andrade M, Malkasian GD, Melton LJ 3rd. Survival patterns after oophorectomy in premenopausa women: a population-based cohort study. Lancet Oncol 2006;7:821-8.

90. Parker WH, Feskanich D, Broder MS, Chang E, Shoupe D, Farquhar CM, et al. Long-term mortality associated with oophorectomy compared with ovarian conservation in the nurses' health study. Obstet Gynecol.2013;121:709-16.

91. Kwon JS, Tinker A, Pansegrau G, McAlpine J, Housty M, McCullum M, et al. Prophylactic salpingectomy and delayed oophorectomy as an alternative for BRCA mutation carriers. Obstet Gynecol.2013;121:14-24.

92. Dell' Anna T, Signorelli M, Benedetti-Panici P, Maggioni a, Fossati R, Fruscio R, et al. Systematic lymphadenectomy in ovarian cancer at second-look surgery: a randomised clinical trial. Br J Cancer [Internet]. 2012 [visitado 2015 Sept 9]; 107:785-92. Disponible en: http://www. pubmedcentral.nih.gov/articlerender.fcgi? artid=34259 68\&tool $=$ pmcentrez\&rendertype $=$ abstract

93. Lawrie T, Medeiros LRF, Rosa DD, Bozzetti MC, Rosa MINES, Edelweiss MI, et al. Laparoscopy versus laparotomy for FIGO Stage I ovarian cancer. Cochrane Database Syst Rev. 2008;(4).

94. Gao J, Yang X, Zhang Y. Systematic lymphadenectomy in the treatment of epithelial ovarian cancer: a metaanalysis of multiple epidemiology studies. Jpn J Clin Oncol [Internet]. 2014 [visitado 2015 Sept 9]; 45:4960. Disponible en: http://jjco.oxfordjournals.org/cgi/ doi/10.1093/jjco/hyu 175 
95. Winter-Roach BA, Kitchener HC, Lawrie TA. Adjuvant (post-surgery) chemotherapy for early stage epithelial ovarian cancer. Cochrane database Syst Rev. 2012;(3):CD004706.

96. Kim HS, Ju W, Jee BC, Kim YB, Park NH, Song YS, et al. Systematic lymphadenectomy for survival in epithelial ovarian cancer: a meta-analysis. Int J Gynecol Cancer. 2010;20:520-8.

97. Maggioni A, Benedetti Panici P, Dell'Anna T, Landoni F, Lissoni A, Pellegrino A, et al. Randomised study of systematic lymphadenectomy in patients with epithelial ovarian cancer macroscopically confined to the pelvis. Br J Cancer. 2006;95:699-704.

98. Yokoyama Y, Sakamoto T, Sato S, Saito Y. Evaluation of cytoreductive surgery with pelvic and paraaortic lymphadenectomy and intermittent cisplatin-based combination chemotherapy for improvement of long-term survival in ovarian cancer. Eur J Gynaecol Oncol. 1999;20:361-6.

99. Chan JK, Munro EG, Cheung MK, Husain A, Teng NN, Berek JS, et al. Association of lymphadenectomy and survival in stage I ovarian cancer patients. Obstet Gynecol. 2007;109:12-9.

100. Vergote I, Du Bois A, Amant F, Heitz F, Leunen K, Harter P. Neoadjuvant chemotherapy in advanced ovarian cancer: On what do we agree and disagree? Gynecol Oncol. 2013;128:6-11.

101. Morrison J, Haldar K, Kehoe S, Ta L. Chemotherapy versus surgery for initial treatment in advanced ovarian epithelial cancer (Review). Cochrane Database Syst Rev. 2014;(8. Art.No.: CD005343).

102. Ghezzi F, Cromi A, Uccella S, Bergamini V, Tomera S, Franchi M, et al. Laparoscopy versus laparotomy for the surgical management of apparent early stage ovarian cancer. Gynecol Oncol. 2007;105:409-13.

103. Lécuru F, Desfeux P, Camatte S, Bissery A, Blanc B, Querleu D. Impact of initial surgical access on staging and survival of patients with stage I ovarian cancer. Int J Gynecol Cancer. 2006;16:87-94.

104. Park J-Y, Bae J, Lim MC, Lim SY, Seo S-S, Kang S, et al. Laparoscopic and laparotomic staging in stage I epithelial ovarian cancer: a comparison of feasibility and safety. Int J Gynecol Cancer. 2008;18:1202-9.
105. Romagnolo C, Gadducci A, Sartori E, Zola P, Maggino T. Management of borderline ovarian tumors: results of an Italian multicenter study. Gynecol Oncol. 2006;101:255-60.

106. Park JY, Kim DY, Kim JH, Kim YM, Kim YT, Nam JH. Surgical management of borderline ovarian tumors: The role of fertility-sparing surgery. Gynecol Oncol. 2009;113:75-82.

107. Desfeux P, Camatte S, Chatellier G, Blanc B, Querleu D, Lécuru F. Impact of surgical approach on the management of macroscopic early ovarian borderline tumors. Gynecol Oncol. 2005;98:390-5.

108. Park HJ, Kim DW, Yim GW, Nam EJ, Kim S, Kim YT. Staging laparoscopy for the management of earlystage ovarian cancer: a metaanalysis. Am J Obstet Gynecol. 2013;209:58.e1-8.

109. Fagotti A, Fanfani F, Ludovisi M, Lo Voi R, Bifulco G, Testa AC, et al. Role of laparoscopy to assess the chance of optimal cytoreductive surgery in advanced ovarian cancer: a pilot study. Gynecol Oncol. 2005;96:729-35.

110. Fagotti A, Ferrandina G, Fanfani F, Garganese G, Vizzielli G, Carone V, et al. Prospective validation of a laparoscopic predictive model for optimal cytoreduction in advanced ovarian carcinoma. Am J Obstet Gynecol. 2008;199:642.e1-6.

111. Fagotti A, Vizzielli G, Fanfani F, Costantini B, Ferrandina $\mathrm{G}$, Gallotta V, et al. Introduction of staging laparoscopy in the management of advanced epithelial ovarian, tubal and peritoneal cancer: impact on prognosis in a single institution experience. Gynecol Oncol. 2013;131:341-6.

112. Covens AL, Dodge JE, Lacchetti C, Elit LM, Le T, Devries-Aboud M, et al. Surgical management of a suspicious adnexal mass: A systematic review. Gynecol Oncol. 2012;126:149-56.

113. Brockbank EC, Harry V, Kolomainen D, Mukhopadhyay D, Sohaib a., Bridges JE, et al. Laparoscopic staging for apparent early stage ovarian or fallopian tube cancer. First case series from a UK cancer centre and systematic literature review. Eur J Surg Oncol. 2013;39:912-7. 
114. Berek JS, Hacker NF. Gynecologic Oncology. 5th edition. Philadelphia: Lipincott Williams \& Wilkins; 2010.

115. Timmers PJ, Zwinderman K, Coens C, Vergote I, Trimbos JB. Lymph Node Sampling and Taking of Blind Biopsies Are Important Elements of the Surgical Staging of Early Ovarian Cancer. Int J Gynecol Cancer. 2010;20:1142-7.

116. García-Soto AE, Boren T, Wingo SN, Heffernen T, Miller DS. Is comprehensive surgical staging needed for thorough evaluation of early-stage ovarian carcinoma? Am J Obstet Gynecol. 2012;206:242.e1-5.

117. Shroff R, Brooks R a, Zighelboim I, Powell M a, Thaker PH, Mutch DG, et al. The Utility of Peritoneal Biopsy and Omentectomy in the Upstaging of Apparent Early Ovarian Cancer. Int J Gynecol Cancer. 2011;21:1.

118. Elias KM, Labidi-Galy SI, Vitonis AF, Hornick JL, Doyle LA, Hirsch MS, et al. Prior appendectomy does not protect against subsequent development of malignant or borderline mucinous ovarian neoplasms. Gynecol Oncol. 2014 132:328-33.

119. Feigenberg T, Covens A, Ghorab Z, Ismiil N, Dubé V, Saad RS, et al. Is routine appendectomy at the time of primary surgery for mucinous ovarian neoplasms beneficial? Int J Gynecol cancer off J Int Gynecol Cancer Soc. 2013;23:1205-9.

120. Lin JE, Seo S, Kushner DM, Rose SL. The role of appendectomy for mucinous ovarian neoplasms. Am J Obstet Gynecol. 2013;208:46.e1-4.

121. Ramírez PT, Slomovitz BM, McQuinn L, Levenback C, Coleman RL. Role of appendectomy at the time of primary surgery in patients with early-stage ovarian cancer. Gynecol Oncol. 2006;103:888-90.

122. Guvenal T, Dursun P, Hasdemir PS, Hanhan M, Guven S, Yetimalar H, et al. Effect of surgical staging on 539 patients with borderline ovarian tumors: A Turkish Gynecologic Oncology Group study. Gynecol Oncol. 2013;131:546-50.

123. Benacerraf BR, Finkler NJ, Wojciechowski C, RC K. Sonographic accuracy in the diagnosis of ovarian masses. J Reprod Med. 1990;35:491-5.
124. Schilder JM, Thompson AM, DePriest PD, et al. Outcome of reproductive age women with stage IA or IC invasive epithelial ovarian cancer treated with fertility - sparing therapy. Gynecol Oncol. 2002;87:1-7.

125. Ayhan A, Celik H, Taskiran C, et al. Oncologic and reproductive outcome after fertility - saving surgery in ovarian cancer. Eur J Gynaecol Oncol 2003;24:223-32.

126. Collinson F, Qian W, Fossati R, Lissoni A, Williams C, Parmar M, et al. Optimal treatment of early-stage ovarian cancer. Ann Oncol. 2014;25:1165-71.

127. Faluyi O, Mackean M, Gourley C, Bryant A, Dickinson HO. Interventions for the treatment of borderline ovarian tumors. Cochrane Database Syst Rev. 2010;(9):CD007696.

128. Morice P, Denschlag D, Rodolakis A, Reed N, Schneider A, Kesic V, et al. Recommendations of the Fertility Task Force of the European Society of Gynecologic Oncology about the conservative management of ovarian malignant tumors. Int J Gynecol Cancer. 2011;21:951-63.

129. Messalli EM, Grauso F, Balbi G, Napolitano A, Seguino E, Torella M. Borderline ovarian tumors: Features and controversial aspects. Eur J Obstet Gynecol Reprod Biol. 2013;167:86-9.

130. Yinon Y, Beiner ME, Gotlieb WH, Korach Y, Perri T, Ben-Baruch G. Clinical outcome of cystectomy compared with unilateral salpingo-oophorectomy as fertility-sparing treatment of borderline ovarian tumors. Fertil Steril. 2007;88:479-84

131. Lenhard MS, Mitterer S, Kümper C, Stieber P, Mayr D, Ditsch N, Friese K, Burges A. Long-term follow-up after ovarian borderline tumor: relapse and survival in a large patient cohort. Eur J Obstet Gynecol Reprod Biol. 2009;145:189-94.

132. Park JY, Kim DY, Kim JH, Kim YM, Kim KR, Kim YT, et al. Micropapillary pattern in serous borderline ovarian tumors: does it matter? Gynecol Oncol. 2011;123:511-6.

133. Kleppe M, Bruls J, van Gorp T, Massuger L, Slangen BFM, van de Vijver KK, et al. Mucinous borderline 
tumors of the ovary and the appendix: A retrospective study and overview of the literature. Gynecol Oncol. 2014;133:155-8.

134. Colombo N, Parma G, Lapresa MT, Maggi F, Piantanida P, Maggioni a. Role of conservative surgery in ovarian cancer: the European experience. Int J Gynecol Cancer. 2005;15(Suppl 3):206-11.

135. Marpeau O, Schilder J, Zafrani Y, Uzan C, Gouy S, Lhommé C, et al. Prognosis of patients who relapse after fertility-sparing surgery in epithelial ovarian cancer. Ann Surg Oncol. 2008;15:478-83.

136. Schilder JM, Thompson AM, DePriest PD, Ueland FR, Cibull ML, Kryscio RJ, et al. Outcome of reproductive age women with stage IA or IC invasive epithelial ovarian cancer treated with fertility-sparing therapy. Gynecol Oncol. 2002;87:1-7.

137. Schlaerth AC, Chi DS, Poynor EA , Barakat RR, Brown CL. Long-term survival after fertility-sparing surgery for epithelial ovarian cancer. Int J Gynecol Cancer. 2009;19:1199-204.

138. Satoh T, Hatae M, Watanabe Y, Yaegashi N, Ishiko O, Kodama S, et al. Outcomes of fertility-sparing surgery for stage I epithelial ovarian cancer: A proposal for patient selection. J Clin Oncol. 2010;28:1727-32.

139. Kajiyama H, Shibata K, Mizuno M, Umezu T, Suzuki S, Nawa A, et al. Long-term survival of young women receiving fertility-sparing surgery for ovarian cancer in comparison with those undergoing radical surgery. Br J Cancer. Nature Publishing Group; 2011;105:1288-94.

140. Wright JD, Shah M, Mathew L, Burke WM, Culhane J, Goldman N, et al. Fertility preservation in young women with epithelial ovarian cancer. Cancer. 2009;115:4118-26.

141. Kajiyama H, Shibata K, Suzuki S, Ino K, Nawa A, Kawai M, et al. Fertility-sparing surgery in young women with invasive epithelial ovarian cancer. Eur J Surg Oncol. 2010;36:404-8.

142. Eisenhauer EL, Abu-Rustum NR, Sonoda Y, Levine DA, Poynor EA, Aghajanian C, et al. The addition of extensive upper abdominal surgery to achieve optimal cytoreduction improves survival in patients with stages IIIC-IV epithelial ovarian cancer. Gynecol Oncol. 2006;103:1083-90.
143. Ang C, Chan KKL, Bryant A, Naik R, Dickinson HO. Ultra-radical (extensive) surgery versus standard surgery for the primary cytoreduction of advanced epithelial ovarian cancer. Cochrane database Syst Rev. 2011;(4):CD007697.

144. Gerestein CG, Damhuis RAM, Burger CW, Kooi GS. Postoperative mortality after primary cytoreductive surgery for advanced stage epithelial ovarian cancer: a systematic review. Gynecol Oncol. 2009;114:523-7.

145. Chi DS, Eisenhauer EL, Lang J, Huh J, Haddad L, Abu-Rustum NR, et al. What is the optimal goal of primary cytoreductive surgery for bulky stage IIIC epithelial ovarian carcinoma (EOC)? Gynecol Oncol. 2006;103:559-64.

146. Wimberger P, Lehmann N, Kimmig R, Burges A, Meier W, Du Bois A. Prognostic factors for complete debulking in advanced ovarian cancer and its impact on survival. An exploratory analysis of a prospectively randomized phase III study of the Arbeitsgemeinschaft Gynaekologische Onkologie Ovarian Cancer Study Group (AGO-OVA. Gynecol Oncol. 2007;106:69-74.

147. Du Bois A, Reuss A, Pujade-Lauraine E, Harter P, Ray-Coquard I, Pfisterer J. Role of surgical outcome as prognostic factor in advanced epithelial ovarian cancer: a combined exploratory analysis of 3 prospectively randomized phase 3 multicenter trials: by the Arbeitsgemeinschaft Gynaekologische Onkologie Studiengruppe Ovarialkarzin. Cancer. 2009;115:1234-44.

148. Peiretti M, Zanagnolo V, Aletti GD, Bocciolone L, Colombo N, Landoni F, et al. Role of maximal primary cytoreductive surgery in patients with advanced epithelial ovarian and tubal cancer: Surgical and oncological outcomes. Single institution experience. Gynecol Oncol. 2010;119:259-64.

149. Wright JD, Lewin SN, Deutsch I, Burke WM, Sun $\mathrm{X}$, Neugut AI, et al. Defining the limits of radical cytoreductive surgery for ovarian cancer. Gynecol Oncol. 2011;123:467-73.

150. Elattar A, Bryant A, Winter-Roach BA, Hatem M, Naik R. Optimal primary surgical treatment for advanced epithelial ovarian cancer. Cochrane database Syst Rev. 2011;(8):CD007565. 
151. Chang SJ, Hodeib M, Chang J, Bristow RE. Survival impact of complete cytoreduction to no gross residual disease for advanced-stage ovarian cancer: a metaanalysis. Gynecol Oncol. 2013;130:493-8.

152. Bristow RE, Puri I, Chi DS. Cytoreductive surgery for recurrent ovarian cancer: a meta-analysis. Gynecol Oncol. 2009;112:265-74.

153. Al Rawahi T, Lopes AD, Bristow RE, Bryant A, Elattar A, Chattopadhyay S, et al. Surgical cytoreduction for recurrent epithelial ovarian cancer. Cochrane Database Syst Rev. 2013;2:CD008765.

154. Shih KK, Chi DS. Maximal cytoreductive effort in epithelial ovarian cancer surgery. J Gynecol Oncol. 2010;21:75-80.

155. Schorge JO, Wingo SN, Bhore R, Heffernan TP, Lea JS. Secondary cytoreductive surgery for recurrent platinum-sensitive ovarian cancer. Int J Gynecol Obstet. 2010;108:123-7.

156. Chi DS, McCaughty K, Diaz JP, Huh J, Schwabenbauer S, Hummer AJ, et al. Guidelines and selection criteria for secondary cytoreductive surgery in patients with recurrent, platinum-sensitive epithelial ovarian carcinoma. Cancer. 2006;106:1933-9.

157. Sehouli J, Richter R, Braicu EI, Bühling KJ, Bahra M, Neuhaus P, et al. Role of secondary cytoreductive surgery in ovarian cancer relapse: who will benefit? A systematic analysis of 240 consecutive patients. J Surg Oncol. 2010;102:656-62.

158. Onda T, Yoshikawa H, Yasugi T, Yamada M, Matsumoto K, Taketani Y. Secondary cytoreductive surgery for recurrent epithelial ovarian carcinoma: proposal for patients selection. Br J Cancer. 2005;92:1026-32.

159. Chi DS, Musa F, Dao F, Zivanovic O, Sonoda Y, Leitao MM, et al. An analysis of patients with bulky advanced stage ovarian, tubal, and peritoneal carcinoma treated with primary debulking surgery (PDS) during an identical time period as the randomized EORTCNCIC trial of PDS vs neoadjuvant chemotherapy (NACT). Gynecol Oncol. 2012;124:10-4.

160. Kang S, Nam B-H. Does neoadjuvant chemotherapy increase optimal cytoreduction rate in advanced ovarian cancer? Meta-analysis of 21 studies. Ann Surg Oncol. 2009;16:2315-20.
161. Tangitgamol S, Manusirivithaya S, Laopaiboon M, Lumbiganon P, Bryant A. Interval debulking surgery for advanced epithelial ovarian cancer. Cochrane Database Syst Rev. 2013;(4):CD006014.

162. Nezhat FR, Pejovic T, Finger TN, Khalil SS. Role of Minimally Invasive Surgery in Ovarian Cancer. J Minim Invasive Gynecol. 2013;20:754-65.

163. Jaaback K, Johnson N, Lawrie T. Intraperitoneal chemotherapy for the initial management of primary epithelial ovarian cancer. Cochrane Database Syst Rev. 2011;(11):CD005340.

164. Elit L, Oliver TK, Covens A, Kwon J, Fung MF-K, Hirte HW, et al. Intraperitoneal chemotherapy in the first-line treatment of women with stage III epithelial ovarian cancer: a systematic review with metaanalyses. Cancer. 2007;109:692-702.

165. Hess LM, Benham-Hutchins M, Herzog TJ, Hsu CH, Malone DC, Skrepnek GH, et al. A meta-analysis of the efficacy of intraperitoneal cisplatin for the front-line treatment of ovarian cancer. Int J Gynecol Cancer. 2007; 17:561-70.

166. Armstrong DK, Bundy B, Wenzel L, Huang HQ, Baergen R, et al. Intraperitoneal Cisplatin and Paclitaxel in Ovarian Cancer. N Engl J Med. 2006;354:34-43.

167. Chiva LM, Gonzalez-Martin A. A critical appraisal of hyperthermic intraperitoneal chemotherapy (HIPEC) in the treatment of advanced and recurrent ovarian cancer. Gynecol Oncol. 2015;136:130-5.

168. Chua TC, Robertson G, Liauw W, Farrell R, Yan TD, Morris DL. Intraoperative hyperthermic intraperitoneal chemotherapy after cytoreductive surgery in ovarian cancer peritoneal carcinomatosis: systematic review of current results. J Cancer Res Clin Oncol. 2009;135:1637-45.

169. Spiliotis J, Halkia E, Lianos E, Kalantzi N, Grivas A, Efstathiou E, et al. Cytoreductive Surgery and HIPEC in Recurrent Epithelial Ovarian Cancer: A Prospective Randomized Phase III Study. Ann Surg Oncol. 2015;22:1570-5. doi: 10.1245/s10434-0144157-9. Epub 2014 Nov 13.

170. Rustin GJ, van der Burg ME, Griffin CL, Guthrie D, Lamont A, Jayson GC, et al. Early versus delayed treatment of relapsed ovarian cancer (MRC OV05/ 
EORTC 55955): a randomised trial. Lancet. 2010; 376:1155-63.

171. Clarke T, Galaal K, Bryant A, Naik R. Evaluation of follow - up strategies for patients with epithelial ovarian cancer following completion of primary treatment. Cochrane Database of Systematic Reviews 2014, Issue 9. Art No. CD006119.

172. Chen YM, Chen T, Zee CS, Shi YP, Wan LR, Tong LJ. Is there an impact of 18F-FDG PET/CT on the surveillance and clinical management of recurrent ovarian cancer? Research based on a large sample in a single PET/CT center. Nucl Med Commun. 2014; $35: 347-52$

173. Gu P, Pan LL, Wu SQ, Sun L, Huang G. CA 125, PET alone, PET-CT, CT and MRI in diagnosing recurrent ovarian carcinoma: a systematic review and metaanalysis. Eur J Radiol. 2009;71:164-74.

174. Limei Z, Yong C, Yan X, Shuai T, Jiangyan X, Zhiqing L. Accuracy of positron emission tomography/ computed tomography in the diagnosis and restaging for recurrent ovarian cancer: a meta-analysis. Int J Gynecol Cancer. 2013; 23:598-607.

175. Kim CY, Jeong SY, Chong GO, Son SH, Jung JH, Kim DH, et al. Quantitative metabolic parameters measured on F-18 FDG PET/CT predict survival after relapse in patients with relapsed epithelial ovarian cancer. Gynecol Oncol. 2015;136:498-504.

176. Rustin GJ, van der Burg ME, MRC obo, EORTC Collaborators. A randomized trial in ovarian cancer (OC) of early treatment of relapse based on CA125 level alone versus delayed treatment based on conventional clinical indicators (MRC OV05/EORTC 55955 trials) [abstract]. J Clin Oncol 2009;27(Suppl 18S): Abstract.

177. Kew F, Galaal K, Bryant A, Naik R. Evaluation of follow-up strategies for patients with epithelial ovarian cancer following completion of primary treatment. Cochrane Database of Systematic Reviews 2011, Issue 6.

178. Geurts SME, de Vegt F, van Altena AM, van Dijck JAAM, Tjan-Heijnen VCG, Verbeek ALM, et al. Considering early detection of relapsed ovarian cancer: a review of the literature. Int J Gynecol Cancer. 2011;21:837-45.

179. Lajer H, Jensen MB, Kilsmark J, Albaek J, Svane D, Mirza MR, et al. The value of gynecologic cancer follow-up: evidence-based ignorance? Int J Gynecol Cancer 2010;20:1307-20.

180. National Comprehensive Cancer Network. Palliative Care. Version 2.2013.

181. Feuer DJ, Broadley KE. Corticosteroids for the resolution of malignant bowel obstruction in advanced gynaecological and gastrointestinal cancer. Cochrane Database of Syst Rev. 2000: CD001219.

182. Ketcham AS, Hoye RC, Pilch YH, Morton DL. Delayed intestinal obstruction following treatment for cancer. Cancer. 1970;25:406-10.

183. Rubin SC, Hoskins WJ, Benjamin I, Lewis JL Jr. Palliative surgery for intestinal obstruction in advanced ovarian cancer. Gynecol Oncol. 1989;34:16-9.

184. van Ooijen B, van der Burg ME, Planting AS, Siersema PD, Wiggers T. Surgical treatment or gastric drainage only for intestinal obstruction in patients with carcinoma of the ovary or peritoneal carcinomatosis of other origin. Surg Gynecol Obstet. 1993;176:469-74

185. Lau PW, Lorentz TG. Results of surgery for malignant bowel obstruction in advanced, unresectable, recurrent colorectal cancer. Dis Colon Rectum. 1993;36:61-4.

186. Feuer DJ, Broadley KE, Shepherd JH, Barton DP. Surgery for the resolution of symptoms in malignant bowel obstruction in advanced gynaecological and gastrointestinal cancer. Cochrane Database Syst Rev. 2000;(4):CD002764. 


\section{Tabla 1. \\ Clasificación para cáncer de ovario, trompa de Falopio o peritoneo (FIGO 2014)}

\section{Estado I}

Compromiso tumoral solamente dentro del ovario (u ovarios) o la(s) trompa(s) de Falopio. No se ha propagado a órganos ni tejidos en el abdomen o la pelvis, ganglios linfáticos ni a lugares distantes.

Estado IA (T1a, N0, M0): compromiso tumoral en un ovario, y el tumor está restringido al interior del ovario; o el tumor se ha desarrollado en una trompa de Falopio, y solo se encuentran dentro de la trompa de Falopio. No hay tumor en la superficie externa del ovario o de la trompa de Falopio. En los exámenes de laboratorio de las muestras de lavado del abdomen y la pelvis no se encontró ninguna célula cancerosa.

Estado IB (T1b, N0, M0): el tumor está presente en ambos ovarios o en las trompas de Falopio, pero no en sus superficies externas. En los exámenes de laboratorio de las muestras de lavado del abdomen y la pelvis no se encontró ninguna célula cancerosa.

Estado IC (T1c, N0, M0): el tumor está presente en uno o ambos ovarios o en las trompas de Falopio, y tiene cualquiera de las siguientes características:

IC1: El tejido (cápsula) que rodea el tumor se reventó durante la cirugía.

IC2: El tumor se encuentra en la superficie exterior de al menos uno de los ovarios o las trompas de Falopio o la cápsula se reventó antes de la cirugía.

IC3: En el examen de laboratorio se encontraron células cancerosas en el líquido o en las muestras de lavado del abdomen.

\section{Estado II}

Compromiso de uno o ambos ovarios o en las trompas de Falopio y se ha propagado a otros órganos en la pelvis.

No se ha propagado a los ganglios linfáticos ni a sitios distantes.

Estado IIA (T2a, N0, M0): el tumor que se originó en los ovarios o las trompas de Falopio se ha propagado o ha invadido al útero, o a las trompas de Falopio o a ambos órganos.

Estado IIB (T2b, N0, M0): el tumor se ha extendido hacia otros tejidos pélvicos intraperitoneales.

\section{Estado III}

Compromiso de uno o ambos ovarios o en las trompas de Falopio, o cáncer peritoneal primario, con compromiso histológico o citológico confirmado a peritoneo fuera de la pelvis o metástasis a ganglios linfáticos retroperitoneales.

Estado IIIA1 (T1 o T2, N1, M0): únicamente ganglios linfáticos retroperitoneales (prueba citológica o histológica): IIIA1(i): metástasis hasta $10 \mathrm{~mm}$ en la mayor dimensión.

IIIA1(ii): metástasis mayores de $10 \mathrm{~mm}$ en la mayor dimensión.

Estado IIIA2 (T3a2, N0 o N1, M0): compromiso peritoneal extrapélvico microscópico (por encima del borde pélvico) con o sin nódulos linfáticos retroperitoneales positivos.

Estado IIIB (T3b, N0 o N1, M0): metástasis peritoneal macroscópica por fuera de la pelvis hasta $2 \mathrm{~cm}$ en la dimensión mayor, con o sin metástasis a ganglios linfáticos retroperitoneales.

Estado IIIC (T3c, N0 o N1, M0): metástasis peritoneal macroscópica por fuera de la pelvis, mayor a 2 cm en la dimensión mayor, con o sin metástasis a ganglios linfáticos retroperitoneales. Incluye extensión del tumor a la cápsula del hígado o bazo sin compromiso parenquimatoso de ambos órganos.

\section{Estado IV (cualquier T, cualquier N, M1)}

Metástasis distantes, excluyendo metástasis peritoneales.

Estado IVA: efusión pleural con citología positiva.

Estado IVB: metástasis parénquima hepático y metástasis a órganos extraabdominales (incluidos ganglios linfáticos inguinales y ganglios linfáticos por fuera de la cavidad abdominal). 


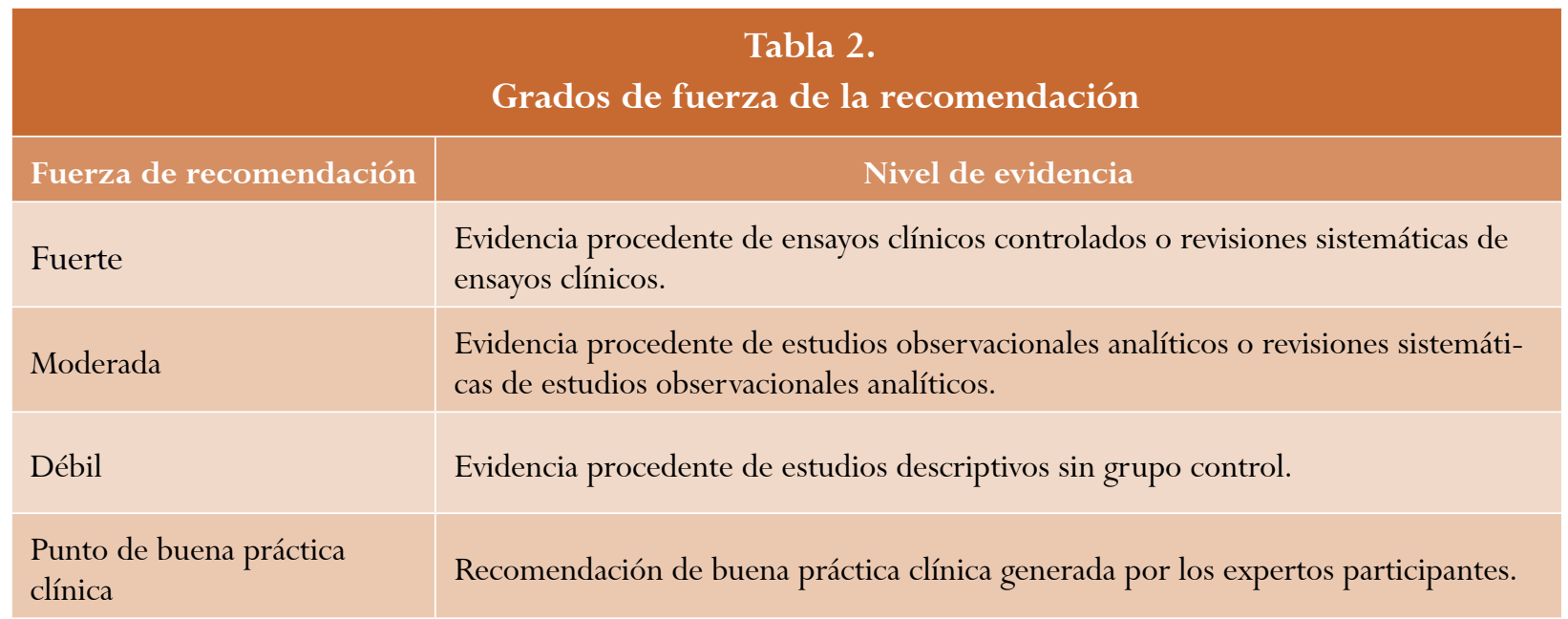

\begin{tabular}{|c|c|}
\hline Fuerza de la recomendación & Recomendación \\
\hline Punto de buena práctica clínica & $\begin{array}{l}\text { A toda paciente con presencia de ascitis, masa abdominal o masa pélvica palpada en el } \\
\text { examen médico o por la paciente, se debe descartar un cáncer de ovario. }\end{array}$ \\
\hline Moderada & $\begin{array}{l}\text { Frente al hallazgo incidental de cáncer de ovario, se recomienda: } \\
\text { - Registrar cuidadosamente todos los hallazgos en el protocolo intraoperatorio, tomar } \\
\text { una biopsia y enviarla a patología. } \\
\text { - Remitir a la paciente a un equipo multidisciplinario especializado en cáncer ginecoló- } \\
\text { gico. } \\
\text { - Abstenerse de realizar procedimientos con cirugías citorreductoras hasta que la pa- } \\
\text { ciente tenga confirmación histológica y se planee una intervención adecuada para su } \\
\text { diagnóstico histológico específico. }\end{array}$ \\
\hline Moderada & $\begin{array}{l}\text { Se recomienda realizar laparotomía de estadificación exhaustiva, la cual incluye laparoto- } \\
\text { mía mediana para permitir la evaluación detallada del abdomen y la pelvis; histerectomía } \\
\text { abdominal total; salpingooforectomía bilateral y omentectomía infracólica; biopsias de } \\
\text { cualquier depósito peritoneal; biopsias aleatorias del peritoneo pélvico y abdominal; y la } \\
\text { evaluación de ganglios linfáticos retroperitoneales mediante una resección ganglionar en } \\
\text { caso de compromiso macroscópico, o linfadenectomía pélvica y paraaórtica completa en } \\
\text { caso de no encontrar lesión macroscópica, siempre que se logre citorreducción completa } \\
\text { en la cavidad abdominal. } \\
\text { Procedimientos mínimamente invasivos pueden ser utilizados en sospecha de estadios } \\
\text { tempranos en pacientes seleccionadas, y deben ser realizados por ginecólogos oncólogos } \\
\text { con experiencia. }\end{array}$ \\
\hline Moderada & $\begin{array}{l}\text { En estadios tempranos el manejo estándar recomendado es la cirugía, la cual incluye: } \\
\text { - Incisión mediana infraumbilical. } \\
\text { - Toma de muestra de ascitis o en su ausencia lavados peritoneales. } \\
\text { - Histerectomía abdominal + salpingooforectomía bilateral } \\
\text { - Resección de lesiones peritoneales sospechosas o aleatorias en caso de no evidencia de } \\
\text { lesión macroscópica. } \\
\text { - Omentectomía infracólica. } \\
\text { - Linfadenectomía pélvica y paraaórtica. } \\
\text { En pacientes con deseo genésico se recomienda salpingooforectomía unilateral. } \\
\text { La vía laparoscópica puede considerarse para el diagnóstico y la estadificación, y ser parte } \\
\text { del tratamiento en estadios tempranos; sin embargo, son necesarios estudios aleatoriza- } \\
\text { dos y controlados. }\end{array}$ \\
\hline
\end{tabular}




\begin{tabular}{|c|c|}
\hline Fuerza de la recomendación & Recomendación \\
\hline Moderada & $\begin{array}{l}\text { Se recomienda el uso de la biopsia por congelación para confirmar enfermedad maligna } \\
\text { y desarrollar un algoritmo de manejo individual. } \\
\text { _ Se deben considerar las limitaciones de la misma en casos de tumores con bajo } \\
\text { riesgo de malignidad. } \\
\text { _ } \quad \text { Las pacientes que deseen mantener potencialmente la fertilidad deben tener una } \\
\text { evaluación intraoperatoria con biopsia por congelación. } \\
\text { - La cirugía de preservación de la fertilidad se puede realizar (si es técnicamente facti- } \\
\text { ble) si los resultados de la biopsia por congelación son positivos para tumor maligno } \\
\text { de células germinales, cáncer epitelial de ovario estadio I o tumores borderline. } \\
\text { - Se debe evitar hacer procedimientos radicales sin biopsia por congelación en casos } \\
\text { de que exista duda. }\end{array}$ \\
\hline Moderada & $\begin{array}{l}\text { La cirugía conservadora de fertilidad puede tenerse en cuenta en mujeres menores de } \\
40 \text { años que deseen paridad, con estadios IA (grado I y II) y IC (grado 1). } \\
\text { - } \quad \text { La paciente debe recibir información completa del procedimiento y posibles desen- } \\
\text { laces. } \\
\text { _ } \quad \text { Debe incluirse estadificación completa de la enfermedad como parte del procedi- } \\
\text { miento. } \\
\text { - En casos seleccionados se sugiere utilizar la vía laparoscópica. } \\
\text { - Debe considerarse completar cirugía cuando esté satisfecho el deseo de paridad. }\end{array}$ \\
\hline Moderada & $\begin{array}{l}\text { Se recomienda la cirugía como manejo estándar inicial de la paciente con cáncer } \\
\text { epitelial de ovario, con el objetivo de clasificar la enfermedad y lograr una citorreducción } \\
\text { completa. } \\
\text { La cirugía radical debe incluir: histerectomía abdominal, salpingooforectomía bilateral, } \\
\text { omentectomía, linfadenectomía pélvica y paraórtica, resección de superficies peritonea- } \\
\text { les, resecciones intestinales, resecciones hepáticas, cola de páncreas y esplenectomía. } \\
\text { Estas cinco últimas solo en caso de evidencia de tumor macroscópico de estos órganos. }\end{array}$ \\
\hline Moderada & $\begin{array}{l}\text { Se recomienda la citorreducción completa como objetivo quirúrgico, para lo cual se } \\
\text { aconseja el abordaje ultrarradical en pacientes con CEO avanzado. } \\
\text { Se recomienda acceder al abdomen superior como parte del abordaje ultrarradical, para } \\
\text { lo cual es indispensable desarrollar equipos de trabajo multidisciplinarios. }\end{array}$ \\
\hline Fuerte & $\begin{array}{l}\text { Se recomienda hacer linfadenectomía pélvica y paraaórtica en pacientes con cáncer } \\
\text { epitelial de ovario en estadio avanzado solamente cuando se logre la citorreducción } \\
\text { completa o exista evidencia de compromiso ganglionar (imágenes o palpación). }\end{array}$ \\
\hline Moderada & $\begin{array}{l}\text { Se recomienda la citorreducción secundaria en pacientes sensibles al platino que recaen } \\
\text { en forma loco-regional seis meses o más posteriores al tratamiento primario. } \\
\text { El objetivo debe ser la citorreducción completa. }\end{array}$ \\
\hline Punto de buena práctica clínica & $\begin{array}{l}\text { En las pacientes con persistencia, progresión de la enfermedad o toxicidad limitan- } \\
\text { te, debe decidirse si serán sometidas a otras líneas de quimioterapia o se reorienta la } \\
\text { intención del tratamiento hacia el control de los síntomas y cuidados de soporte. Estas } \\
\text { pacientes también podrían incluirse en ensayos clínicos donde estén disponibles. }\end{array}$ \\
\hline
\end{tabular}




\section{Figura 1.}

Manejo quirúrgico integral

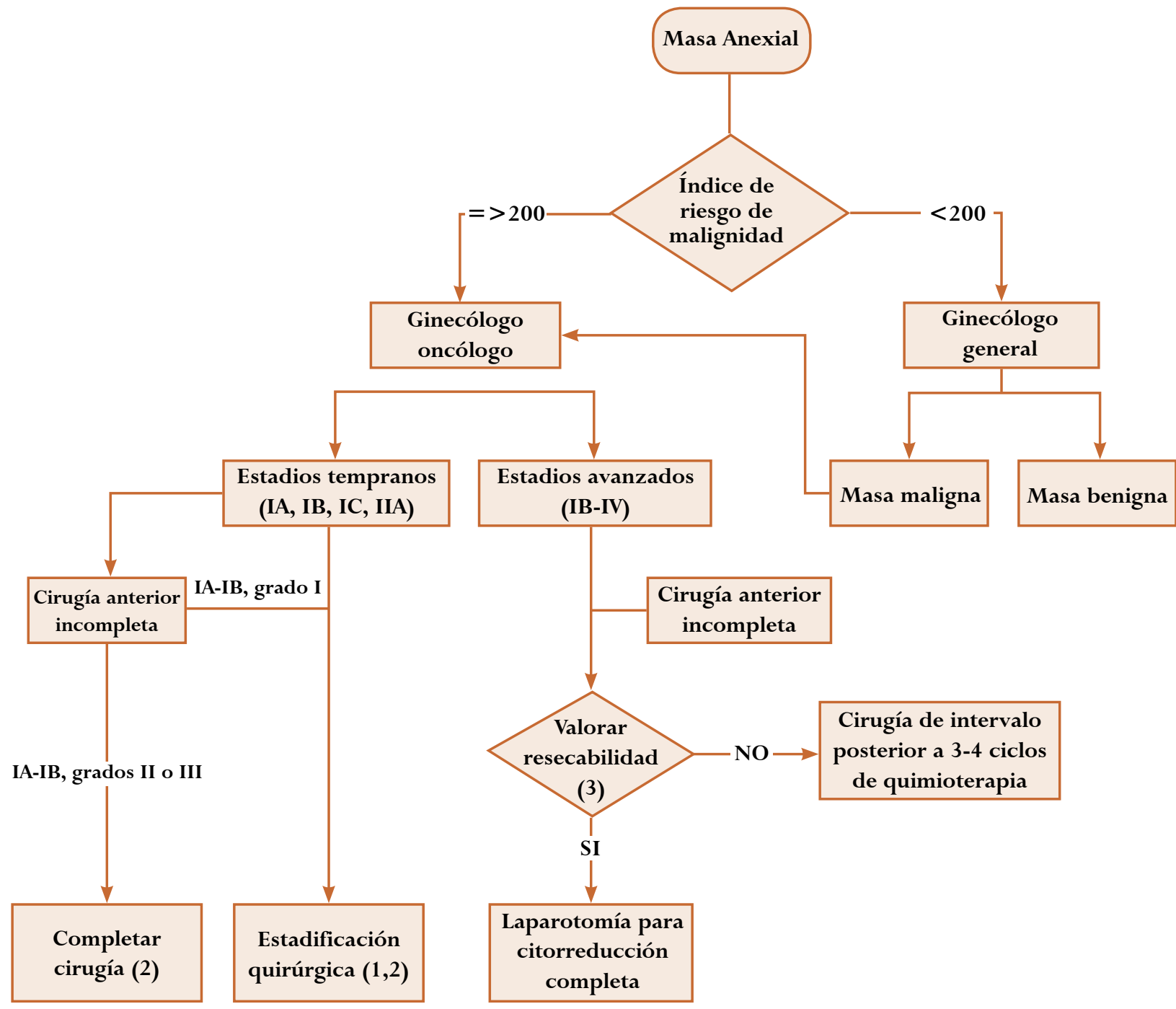

NOTAS

1. La cirugía en estadios tempranos incluye: incisión mediana infraumbilical, toma de muestra de ascitis o, en su ausencia, lavados peritoneales, histerectomía abdominal, salpingooforectomía bilateral, resección de lesiones peritoneales sospechosas o aleatorias en caso de no evidencia de lesión macroscópica, omentectomía infracólica, linfadenectomía pélvica y paraaórtica.

2. Considerar preservación de la fertilidad y cirugía mínimamente invasiva en mujeres que lo manifiesten.

3. La valoración incluye criterios imagenológicos (índice de carcinomatosis peritoneal), laparoscópicos (índice de Fagotti), comorbilidades.

4. Considerar cirugía de intervalo y tres ciclos de quimioterapia.

5. Considerar quimioterapia intraperitoneal. 
Figura 2.

Manejo de tumores de bajo potencial de malignidad

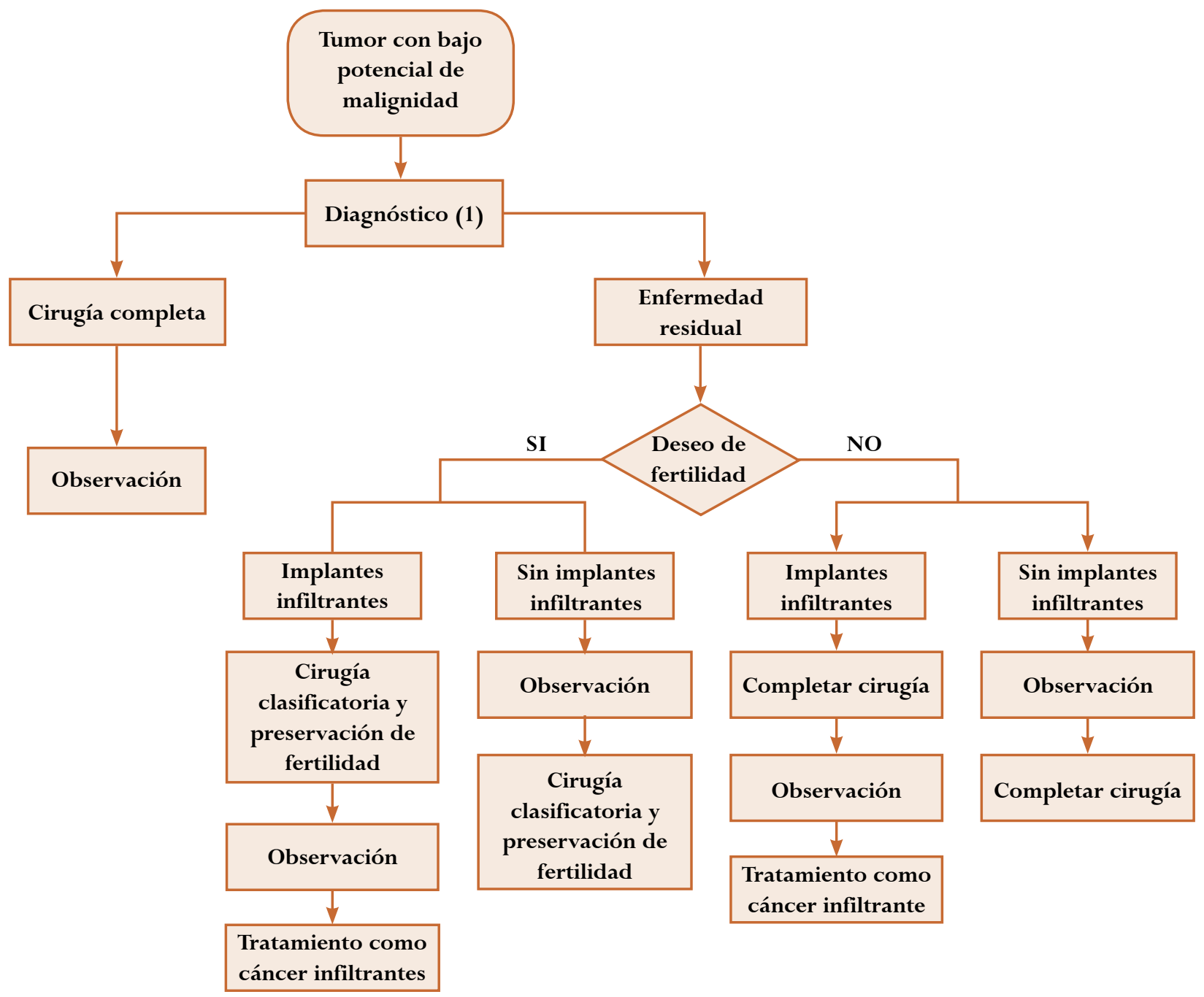


146 Revista Colombiana de Obstetricia y Ginecología Vol. 67 No. 2 • 2016

Anexo 1.

Índice de riesgo de malignidad para la evaluación de pacientes con masas pélvicas

$$
R M I=U \times M \times S \times C A 125
$$

RMI: Índice de riesgo de malignidad

U: $\quad$ Puntuación ecográfica

M: $\quad$ Estado pre y posmenopáusico

T: $\quad$ Tamaño tumoral

CA125: Nivel sérico de CA125

El puntaje se calcula de la siguiente forma:

Puntuación ecográfica: se da un punto por cada uno de los siguientes hallazgos: presencia de lesión cística multilocular, áreas sólidas, lesión bilateral, ascitis o metástasis intraabdominal. Si tiene un valor de 0 o 1 , entonces $U=1$; si tiene un valor mayor o igual a 2, luego $U=2$.

Estado pre y posmenopáusico: estado posmenopáusico es definido como más de un año de amenorrea o edad mayor a 50 años quienes tuvieron histerectomía previa. De lo contrario son consideradas en estado pre menopáusico. Si está en estado premenopáusico, luego $M=1$. Si se encuentra en estado post menopáusico, luego $M=4$.

Tamaño tumoral: tamaño del tumor medido por ecografía sobre el diámetro más grande de la imagen. $S=1$ tamaño menor a $7 \mathrm{~cm} . S=2$ tamaño mayor o igual a $7 \mathrm{~cm}$.

Nivel sérico de CA125: se incluye directamente el valor encontrado. 